Bukow, Wolf-D.

\title{
Anfragen an die Praxis der Sozialen Arbeit in einer von Mobilität und Vielfalt geprägten Postmoderne - Soziale Arbeit in der Migrationsgesellschaft
} Spetsmann-Kunkel, Martin [Hrsg.]; Frieters-Reermann, Norbert [Hrsg.]: Soziale Arbeit in der Migrationsgesellschaft. Opladen ; Berlin ; Toronto : Verlag Barbara Budrich 2013, S. 13-43. - (Schriften der Katholischen Hochschule Nordrhein-Westfalen; 17)

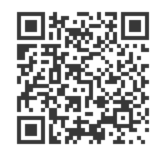

Quellenangabe/ Reference:

Bukow, Wolf-D.: Anfragen an die Praxis der Sozialen Arbeit in einer von Mobilität und Vielfalt geprägten Postmoderne - Soziale Arbeit in der Migrationsgesellschaft - In: Spetsmann-Kunkel, Martin [Hrsg.]; Frieters-Reermann, Norbert [Hrsg.]: Soziale Arbeit in der Migrationsgesellschaft. Opladen ; Berlin ; Toronto : Verlag Barbara Budrich 2013, S. 13-43 - URN: urn:nbn:de:0111-pedocs-127798 DOI: $10.25656 / 01: 12779$

https://nbn-resolving.org/urn:nbn:de:0111-pedocs-127798

https://doi.org/10.25656/01:12779

in Kooperation mit / in cooperation with:

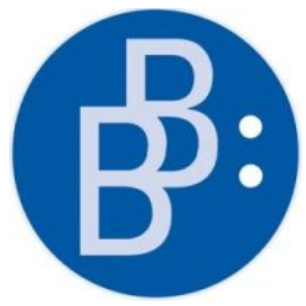

https://www.budrich.de

\section{Nutzungsbedingungen}

Dieses Dokument steht unter folgender Creative Commons-Lizenz: http://creativecommons.org/licenses/by-nc-nd/3.0/de/deed - Sie dürfen das Werk bzw. den Inhalt unter folgenden Bedingungen vervielfältigen, verbreiten und öffentlich zugänglich machen: Sie müssen den Namen des Autors/Rechteinhabers in der von inm festgelegten Weise nennen. Dieses Werk bzw. dieser Inhalt darf nicht für kommerzielle Zwecke verwendet werden und es darf nicht bearbeitet, abgewandelt oder in anderer Weise verändert werden.

Mit der Verwendung dieses Dokuments erkennen Sie die Nutzungsbedingungen an.

\section{Terms of use}

This document is published under following Creative Commons-License: http://creativecommons.org/licenses/by-nc-nd/3.0/de/deed.en - You may copy, distribute and transmit, adapt or exhibit the work in the public as long as you attribute the work in the manner specified by the author or licensor. You are not allowed to make commercial use of the work or its contents. You are not allowed to alter, transform, or change this work in any other way.

By using this particular document, you accept the above-stated conditions of use.

\section{Kontakt / Contact:}

\section{peDOcs}

DIPF | Leibniz-Institut für Bildungsforschung und Bildungsinformation

Informationszentrum (IZ) Bildung

E-Mail: pedocs@dipf.de

Internet: www.pedocs.de

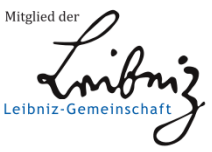


Martin Spetsmann-Kunkel

Norbert Frieters-Reermann (Hrsg.)

\section{Soziale Arbeit in der Migrationsgesellschaft}

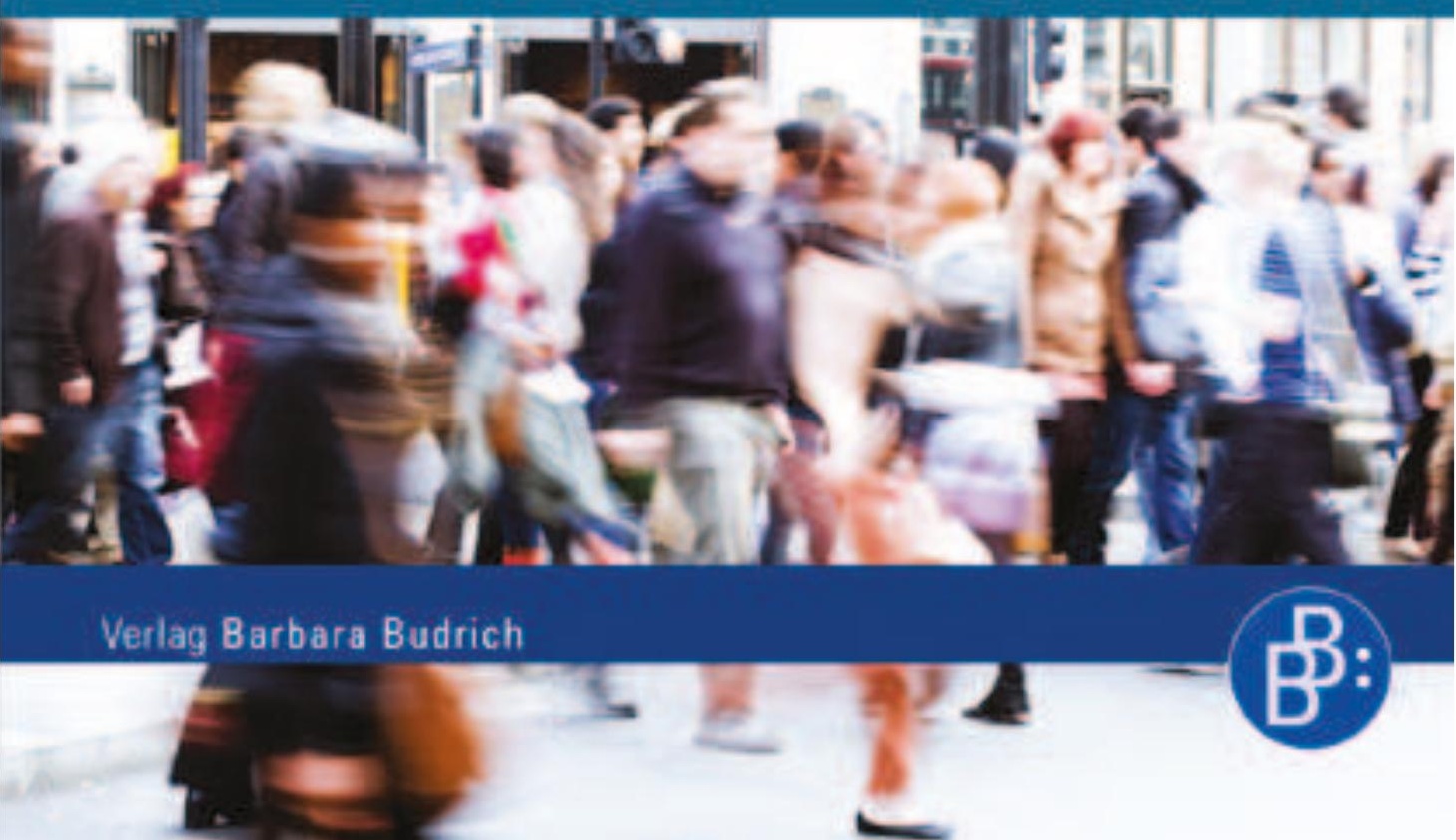


Schriften der Katholischen Hochschule

Nordrhein-Westfalen

Band 17

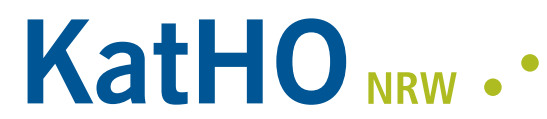

Aachen | Köln | Münster | Paderborn

Katholische Hochschule Nordrhein-Westfalen Catholic University of Applied Sciences 
Martin Spetsmann-Kunkel

Norbert Frieters-Reermann (Hrsg.)

\section{Soziale Arbeit in der} Migrationsgesellschaft

Verlag Barbara Budrich

Opladen, Berlin \& Toronto 2013 
Prof. Dr. Martin Spetsmann-Kunkel

Prof. Dr. Norbert Frieters-Reermann

Katholische Hochschule NRW / Aachen

Robert-Schuman-Straße 25

52066 Aachen

Tel.: (+49) (0)241-6000318

Bibliografische Information der Deutschen Nationalbibliothek

Die Deutsche Nationalbibliothek verzeichnet diese Publikation in der Deutschen

Nationalbibliografie; detaillierte bibliografische Daten sind im Internet über

http://dnb.d-nb.de abrufbar.

(C) 2013 Dieses Werk ist im Verlag Barbara Budrich erschienen und steht unter folgender Creative Commons Lizenz: http://creativecommons.org/licenses/by-nc$\mathrm{nd} / 3.0 / \mathrm{de} /$

Verbreitung, Speicherung und Vervielfältigung erlaubt, kommerzielle Nutzung und Veränderung nur mit Genehmigung des Verlags Barbara Budrich

Dieses Buch steht im OpenAccess Bereich der Verlagsseite zum kostenlosen Download bereit (https://doi.org/10.3224/93809468)

Eine kostenpflichtige Druckversion kann über den Verlag bezogen werden. Die Seitenzahlen in der Druck- und Onlineversion sind identisch.

\section{ISBN 978-3-938094-68-6 (Paperback) \\ eISBN 978-3-8474-0337-1 (eBook) \\ DOI $\quad 10.3224 / 93809468$}

Umschlaggestaltung: disegno visuelle kommunikation, Wuppertal - www.disenjo.de Typografisches Lektorat: Petra Reiners, Bonn - www.buchfinken.com Druck: paper \& tinta, Warschau

Printed in Germany 


\section{Inhaltsverzeichnis}

1. Soziale Arbeit in der Migrationsgesellschaft einleitende Gedanken

Martin Spetsmann-Kunkel

2. Anfragen an die Praxis der Sozialen Arbeit in einer von Mobilität und Vielfalt geprägten Postmoderne - Soziale Arbeit in der Migrationsgesellschaft

Wolf-D. Bukow

3. Interkulturelle Soziale Arbeit: Integration, Anerkennung und Partizipation als Leitideen einer differenzsensiblen Sozialen Arbeit in der Migrationsgesellschaft

Josef Freise

4. Interkulturelles Lernen und Forschen in transkulturellen Dialoggruppen

Cornelia Muth

5. Demokratiepädagogik und Diversity Education pädagogische Konzepte und ihre Bedeutung für die Soziale Arbeit

Schahrzad Farrokhzad 
6. Kritische Soziale Arbeit in Diskriminierungs- und Herrschaftsverhältnissen - eine Skizze

Claus Melter

7. Soziale Arbeit in mobiler Gesellschaft Institutionelle Bedingungen, professionelle Expertise und das eigene Verschränkt-Sein in Alltagsrassismus und kulturalisierenden Deutungsprozessen

Claudia Roller

8. Interkulturelle Öffnung als Team - von Stolpersteinen und Meilensteinen

Norbert Teutenberg

9. Die Migrationssozialarbeit der Caritas

Thomas Kley

10. Soziale Arbeit und Migration - Auslassungen, Anregungen und Ausblicke

Norbert Frieters-Reermann 


\title{
2. Anfragen an die Praxis der Sozialen Arbeit in einer von Mobilität und Vielfalt geprägten Postmoderne - Soziale Arbeit in der Migrationsgesellschaft
}

\author{
Wolf-D. Bukow
}

\section{Vorbemerkung}

Die Praxis der Sozialen Arbeit, der Sozialpädagogik, aber auch der Pädagogik allgemein muss sich auf ein stark verändertes Handlungsfeld und damit einige völlig neue Herausforderungen einstellen. Wir haben es heute mit einem globalgesellschaftlich geprägten urbanen Handlungsfeld $\mathrm{zu}$ tun. Und hier sind es vor allem die durch die technische Entwicklung hervorgerufenen neuen Formen der Mobilität, der Medien und der Märkte, die zu einer erheblichen Zunahme an Diversität, an Fluktuation und Virtualisierung der Orientierungen beigetragen haben. Vor dem Hintergrund dieser massiven Neukonstruktion von gesellschaftlicher Wirklichkeit müssen viele überkommenen Theorien und Methoden sozialer Arbeit und Sozialpädagogik überdacht und neu ausgerichtet werden. Hinzu kommt noch, dass man sich dabei nur wenig auf vorliegendes Wissen verlassen kann. Die einschlägigen Fachwissenschaften sind erst dabei, die Phänomene neu zu interpretieren. Und in der Öffentlichkeit tut man sich noch viel schwerer, die „Zeichen der Zeit" zu deuten und entsprechende Folgerungen zu ziehen. Gerade im Blick auf Mobilität und Diversität ist man eher noch damit beschäftigt, alte Weltbilder zu retten, statt sich auf die veränderten Bedingungen einzustellen.

All dies nötigt dazu, sich mit der gesellschaftlichen Wirklichkeit noch einmal grundsätzlich neu zu befassen und sich auf diese Weise auf die veränderte Situation neu einzustellen. Unterdessen ist der gesellschaftliche Wandel so weit fortgeschritten, dass sogar eine radikale Neuausrichtung des Blicks notwendig ist. Anschließend wäre zu prüfen, was sich daraus für Folgerungen für die Praxis ergeben. So gesehen geht es um drei Schritte:

a) Zunächst muss man sich darüber klar werden, inwiefern eine Neuausrichtung des Blicks nötig ist und was dabei zu berücksichtigen ist.

b) Sodann kommt es darauf an, sich darüber zu verständigen, worin der aktuelle gesellschaftliche Wandel besteht und wie sich vor diesem Hintergrund das soziale Feld gewandelt hat. 
c) Und schließlich wäre zu prüfen, welche Herausforderungen sich daraus für eine entsprechend aktualisierte Soziale Arbeit, hier z.B. für soziale Intervention ergeben.

Damit sind die folgenden Schritte bereits markiert. Sicherlich ist es sinnvoll, sich zunächst mit der Neuausrichtung des Blicks etwas genauer zu befassen, auch wenn man dabei nicht sofort erkennen kann, welche Implikationen das für die Praxis der Sozialen Arbeit hat. Aber von dem gewählten Blickwinkel hängt $a b$, wie man das Handlungsfeld beobachtet und was man dort an Herausforderungen ausmachen kann. Und das ist natürlich entscheidend dafür, welche Handlungsmöglichkeiten sich ergeben.

\section{Inwiefern zunehmende Mobilität und Diversität zum Auslöser für einen neuen Blick auf die gesellschaftliche Wirklichkeit werden}

Die Pädagogik und vor allem die Sozialpädagogik und die Soziale Arbeit sind mit alltäglichen Lebenslagen befasst. Und wenn es um solche Lebenslagen geht, dann muss man sich darüber klar sein, dass damit Menschen in ganz bestimmten, in historisch-konkreten Situationen gemeint sind - in Situationen, die ihnen heute und jetzt eine sinnhaft-soziale Existenz ermöglichen bzw. aus bestimmten Gründen vielleicht auch erschweren oder gar unmöglich machen. Es geht damit genauer formuliert um jemanden innerhalb eines für ihn hier und heute relevanten gesellschaftlichen Referenzrahmens. Diese Betrachtungsweise ist an sich nicht neu; sie ist im Prinzip seit langem vertraut, wird damit allerdings auch für weitgehend abgearbeitet gehalten. Dabei ist die Sensibilität für einen möglichen gesellschaftlichen Wandel verloren gegangen. Tatsächlich erleben wir zurzeit einen massiven und für den Einzelnen hier und heute extrem folgenreichen gesellschaftlichen Wandel, den Übergang von der Moderne zur Postmoderne.

\section{Gesellschaft ist heute primär Stadtgesellschaft}

Die Verortung klingt zunächst trivial, ist jedoch hoch brisant, wenn man sich die Implikationen eines solchen Ansatzes genauer anschaut. Das, was eine Gesellschaft ausmacht, wird hier nicht mehr abstrakt definiert, sondern daran festgemacht, was eine gesellschaftliche Konstruktion in der Praxis des Alltagshandelns konstituiert. Traditioneller Weise denkt man bei Gesellschaft jedoch an eine Konfiguration, die auf einer Augenhöhe mit dem Staat ange- 
siedelt ist, was dazu führt, Gesellschaft, Kultur und Sprache zusammen zu denken. Gesellschaft wird dabei zu so etwas wie ein alles überwölbender Container, in den man einfügt, was die Tradition des Nationalstaates so hergibt. Neben den genannten drei Aspekten sind es dann schnell auch noch Religion und Geschichte und ggf. auch noch Heimat und weitere Accessoires aus dem im 19. Jahrhundert entstandenen „Projekt der Moderne“. Das Problem ist nur, dass diese Vorstellung von Gesellschaft weniger denn je mit der empirischen Praxis zu tun hat. Dieses Konzept war nie sozial-adäquat, schon weil es ordnungspolitisch top down angelegt war. Niemals hat eine von oben „erlassene“ nationale Idee den Einzelnen wirklich eindeutig definiert und geprägt. Vielmehr arrangiert man sich im Alltag schon immer und alternativlos reflexiv zu dem, was „,zuhanden“ ist. Die Basis der Gesellschaft besteht deshalb aus Vielen als Viele. Und wenn man von ihnen in ihrem gesellschaftlichen Kontext ausgeht, dann nimmt die Unschärfe mit der Entfernung immer mehr zu. Je weiter man den Blick erhebt, umso unterschiedlicher ist das, was für den Einzelnen als Einzelnen relevant ist. Bezugsgruppen vermögen da den Blick noch einmal etwas zu bündeln. Bildung mag den Blick noch einmal auf bestimmte Themen fokussieren. Aber wenn schon der Kern der Gesellschaft aus Vielen als Viele besteht, wie heterogen muss dann der Horizont sein, unter dem sich diese bewegen.

Die genannte Verortung muss man spätestens heute neu „lesen“. Tatsächlich richten wir mit dieser Verortung die Aufmerksamkeit einerseits auf den urbanen Alltag und damit auf die Stadtgesellschaft, die den letzten noch face-to-face erlebbaren Horizont des Hier und Heute bildet, und anderseits aber auch auf die Globalgesellschaft, weil alles, was die Stadtgesellschaft überschreitet, sich längst in einem hoch differenzierten und komplex vernetzten globalen Kontext (Bukow 2010a, S. 116ff.) abspielt. Diese Beobachtung bedeutet, dass allem, was Regionen, einzelne Länder, ganze Staaten und sogar supranationale Zusammenschlüsse wie die EU betrifft, nur noch eine relative Bedeutung zukommt. Es bleibt im Einzelfall zu prüfen, inwiefern das, was ein Land oder eine Region betrifft, in der Dynamik zwischen Stadt- und Weltgesellschaft tatsächlich wirksam wird. Es bleibt auch zu prüfen, im Blick auf welche Thematik es überhaupt relevant wird. Wirtschaftliche Prozesse sind anders vernetzt als religiöse Bewegungen, wissenschaftliche Diskurse anders als Sprachgemeinschaften.

Das soziale Feld, das hier interessiert, ist also zunächst einmal die Stadtgesellschaft, aber eine Stadtgesellschaft im globalen Kontext. Wie folgenreich ein derartiger Fokus auf die Stadtgesellschaft in ihrem globalen Kontext ist, das lässt sich am einfachsten an einem jüngst in Köln analysierten Vor- 
fall, nämlich der sogenannten „Kalker Revolte“"1 illustrieren. Dort ging es den demonstrierenden allochthonen Jugendlichen darum, für sich als „Kalker Jungs“ - exemplarisch am Vorfall festgemacht - mehr Gerechtigkeit einzufordern. Der Referenzrahmen für diese Forderung ist, dass sie, die sie in der Stadt aufgewachsen sind, sich als Teil der Stadtgesellschaft betrachten. Die Mittel, um diese Selbsteinschätzung als Forderung publik zu machen, entlehnen sie aus global verbreiteten zivilgesellschaftlichen Praktiken. Die Kommune allerdings betrachtet die allochthone Einwohnerschaft des Stadtquartiers nach wie vor als Ausländer bzw. als Menschen mit Migrationsgeschichte. Damit wird unterstellt, dass sie, nur weil sie selbst oder einer ihrer Vorfahren irgendwann einmal eine Staatsgrenze überschritten haben, innerhalb eines Stadtquartiers nur so etwas wie eine Gastrolle spielen dürfen. Die kommunale Einschätzung geht wie selbstverständlich von einer nationalstaatlichen Zurechnung aus, bei der die Stadt zu einer Kleinausgabe eines Nationalstaates schrumpft. Die nationalstaatliche Zurechnung macht aber nur Sinn, wenn man den Nationalstaat gleichzeitig als Gesellschaft betrachtet. So werden aus der Staatsgrenze Volksgrenzen („Volksgemeinschaftsgrenzen“) und das Überschreiten dieser Grenze zum Eintritt in einen national eindeutigen, in sich sozial, kulturell, religiös, rechtlich usw. geschlossenen Kulturraum. Das impliziert, dass sich jedermann bei der Niederlassung dann diesem Kulturraum einfügen, d.h. sich ihm zu assimilieren hat. Wenn man der oben skizzierten Verortung folgt, dann handelt es sich bei der kommunalen Einschätzung der Situation um eine Zumutung, eine nicht nur historisch überholte, sondern immer schon inadäquate, rein ordnungspolitische Zumutung (Anhorn/Stehr 2012, S. 71f.). Und die Jugendlichen kritisieren die Implikationen exakt dieser Zumutung. Im Grunde macht die Kommune das, was schon seit fast zwanzig Jahren als methodologischer Nationalismus kritisiert wird. ${ }^{2}$

1 Auslöser für die im Januar 2008 über eine Woche andauernden Proteste der Menschen in dem Stadtteil Köln-Kalk war der Tod eines 17 jährigen Jugendlichen aus dem Viertel, der bei einer Auseinandersetzung mit zwei weiteren Jugendlichen aus Köln-Kalk tödlich verletzt wurde. Was sich am 18.01.2008 in Köln-Kalk ereignet hat, lässt sich in wenigen Strichen skizzieren: In einem Gerangel unter Jugendlichen wird ein Schüler erstochen. Die Polizei sagt, Sahli habe ,abziehen' wollen und sei in Notwehr getötet worden. Der beschuldigte Jugendliche ist marokkanischer Herkunft. Der andere, der zum Messer greift, stellt sich später heraus, ist Deutscher - mit russischer Herkunft. Der Fall scheint für die Behörden schon acht Stunden nach der Tat klar, obwohl weder endgültig geklärt wird, ob es wirklich ums Abziehen ging, noch, warum es zu der tödlichen Reaktion kommen musste. Der Vorfall löste in dem Stadtteil die Anteilnahme der Bevölkerung und eine wochenlange Protestwelle aus, an der zum großen Teil Jugendliche des Viertels beteiligt waren (Bukow u.a. 2013).

2 Was den methodologischen Nationalismus betrifft, so hat vor allem Ulrich Beck (Beck/Willms 2001) mit diesem Begriff deutlich machen wollen, dass die politisch erzeugte Basisunterscheidung zwischen national und international als sozialwissenschaftliches 


\section{Mit der Orientierung an der Stadtgesellschaft ändert sich auch die Deutung des sozialen Feldes}

Hier geht es freilich nicht nur darum, eine allochthone Bevölkerungsgruppe neu zuzuordnen. Es geht darum, dass die Effekte von Mobilität im sozialen Feld grundsätzlich anders als bisher beschrieben werden müssen. Natürlich wird jemand, der neu in einer Stadt ist, nicht gleich überall mitspielen können. Er muss sich erst mit vielerlei Dingen bis hin zur lokalen Infrastruktur vertraut machen. Das hat aber nichts mit einer „migrationsspezifischen“ Fremdheit, also der Überwindung einer Staatsgrenze zu tun, sondern ist schlicht die konkrete Auswirkung gelebter Mobilität. Es geht in diesem Fall um das Ankommen und ein Sich-Niederlassen in einer wohlumgrenzten Stadtgesellschaft, wobei es letztlich belanglos ist, wo man sich zuvor aufgehalten hat und ob man gar eine Staatsgrenze überschritten hat. Und noch belangloser ist, wo die Eltern her kommen. Man muss sich in jedem Fall hier und heute neu arrangieren - jeder muss das in so einer Situation. Aber jeder, dem man die Möglichkeit dazu einräumt, schafft das auch in kurzer Zeit. Und wenn er sogar schon im Quartier geboren ist, dann geht es nicht mehr um ein „Hineinwachsen“, sondern umgekehrt um ein „Hinauswachsen“" und ein altersentsprechendes Arrangement innerhalb einer globalisierten Stadtgesellschaft.

Der methodologische Nationalismus verhindert die Sicht darauf, dass es hier und heute darum geht, sich an einem längst globalisierten Ort ,passend“ $\mathrm{zu}$ arrangieren und dazu alles, was lokal bis global zur Hand ist, einzusetzen. Und er verführt dazu, die aus praktizierter Mobilität hervorgebrachte Diversität zu ignorieren, ja zu diskreditieren, statt sie als eine Leistung des Subjekts anzuerkennen. Wenn es dann darum geht, das Engagement als Sozialarbeiter oder Pädagoge neu zu ,adressieren“ und auch die dabei aufscheinenden Herausforderungen situationsgemäß einzuschätzen, dann steht einem der methodologische Nationalismus nicht nur im Sinn einer unangemessenen Zurechnung, einer falsch angelegten Methodik, sondern auch einer unangemessenen Erkenntnisweise, d.h. einer falsch angelegten Hermeneutik im Wege. Im Blick auf eine Soziale Praxis, die auf dem Verstehen des Anderen basiert, wäre folglich von hermeneutischem Nationalismus zu sprechen.

Dass der methodologische Nationalismus sich in der Praxis als hermeneutischer Nationalismus auswirkt, auch das lässt sich an dem angeführten Beispiel gut illustrieren. Es wird nämlich beim „Ausländer“ nicht nur unter-

Instrumentarium nicht taugt. Und genau diese Unterscheidung wird vor allem auch in der Migrations- und Minderheitenforschung genauso wie in der interkulturellen Forschung unreflektiert als leitender Begriffs- und Forschungshorizont verwendet (Pries 2010, S. 19). 
stellt, dass er mit dem Überschreiten der Stadtgrenze seine fremdländische Prägung überwinden muss, sondern eben auch, dass er sich bemühen muss, im überkommenen Sozial-, Kultur-, Religions- und Rechtsverständnis ,,anzukommen", sich einzufügen und zu integrieren. Aus der nationalstaatlichen Definition erwächst eine nationale Hermeneutik, eine Hermeneutik der Integration. Der so ausgerichtete hermeneutische Blick verhindert die Erkenntnis, dass es jemandem, der sich in einer bestimmten Situation arrangiert, ob er nun ein Alteingesessener oder ein neu Hinzugekommener ist, einfach darum geht, seine konkreten Bedürfnisse, Wünsche und Hoffnungen zu befriedigen - und zwar ohne das, was ihm im Verlauf seiner Sozialisation im globalisierten urbanen Quartier wichtig geworden ist, was ihn ausmacht, aufzugeben. Der nationalstaatlich aufgeladene hermeneutische Blick übersieht, dass in einer mobilen Gesellschaft zwangsläufig nur immer und immer wieder gemischte Einstellungen zur Grundlage des individuellen Arrangements werden, also zwangsläufig und unumkehrbar Diversität zur Grundlage von Individualität avanciert. Eine sozialisatorisch vermittelte und verinnerlichte Einstellung und die daraus resultierende persönliche Lebensauffassung sind in einer solchen Situation gerade unabdingbar, sie sind die Motivationsbasis, um sich auf Neues überhaupt einlassen zu können. Und umgekehrt gesehen, basieren die Vorstellungen und Erwartungen hinsichtlich dieser Basisanliegen, die inhaltliche Ausfüllung solcher mit Arbeit, Wohnung, sozialer Sicherheit, Recht, Bildung und Zusammenleben verknüpften „needs“ auf dem je individuell wichtigen konkreten Lebenstil bzw. dem jeweils orientierenden Milieu und den vor Ort erkennbaren individuellen Chancen für eine einem vorschwebende erfolgreiche Lebensführung. Für eine Sozialpädagogik oder eine Sozialarbeit, die sich mit Menschen vor Ort befasst, kommt es demnach darauf an, die Menschen einerseits als Mitglieder einer globalisierten Stadtgesellschaft einzuordnen und anderseits, deren in dieser so geprägten Lebenswelt komponierte konkrete Vorstellungen, Wünsche und Hoffnungen situationsadäquat „lesen“ zu lernen. Dazu muss man sich möglichst genau auf die hier und heute gelebte und zugleich global vernetzte Lebenswelt beziehen.

Das Beispiel belegt, wie zynisch der hermeneutische Nationalismus in der Praxis wirkt. Wenn die allochthonen Jugendlichen, die im Quartier aufgewachsen sind, sich diskriminiert fühlen und deshalb für mehr Gerechtigkeit demonstrieren und dann ihr Anliegen in Verdrehung ihrer Botschaft damit beantwortet wird, dass man ihnen zusätzliche „Integrationsmaßnahmen“ anbietet, so erscheint dieses Angebot vor dem skizzierten Hintergrund schon mehr als nur zynisch. Die ursprüngliche Botschaft der Jugendlichen wird ignoriert, indem man ihr Anliegen nach Form (wir demonstrieren) und Inhalt (wir sind Kalker) zerlegt und damit das Anliegen als solches desavouiert. Anschließend werden sie durch Verweis auf die nationale Herkunft ihrer 
Eltern diskreditiert: Ihr Verhalten signalisiere eine hohe „Ausländer“-Gewaltbereitschaft; ihre Forderungen belegen ihre Unkenntnis über ihre hiesige urbane Situation. Man sei jedoch bereit, ihnen auf die Sprünge zu helfen, damit sie endlich in Deutschland ,ankommen“: Bändigung ihrer Aggression, Unterweisung im hiesigen Lebensstil (vgl. Bukow u.a. 2013).

Will man die Sozialpädagogik oder die Sozialarbeit unter den Bedingungen einer zunehmend globalisierten Stadtgesellschaft neu bestimmen, muss man offensichtlich nicht nur den Blick, sondern auch die daran anschließende Deutung des sozialen Feldes völlig neu ausrichten und darauf achten, die Zusammenhänge so in den Blick zu nehmen, wie sie sich hier und heute tagtäglich mehr oder weniger erfolgreich einspielen, also die Dynamik zwischen „global“ und „lokal“ in den Mittelpunkt zu stellen und von dort her alles entsprechend einzuschätzen, auch mögliche Herausforderungen, Probleme und Verwerfungen situationsadäquat neu „lesen“ lernen.

\section{Es ist wichtig, sich über die gewandelten Bedingungen der Stadtgesellschaft zu verständigen}

In den letzten Jahren hat sich tatsächlich auch empirisch erwiesen, dass es sinnvoll ist, die Stadtgesellschaft als eine nicht nur eigenständige, sondern vor allem emergente soziale Einheit ${ }^{3} \mathrm{zu}$ betrachten. Im Grunde trägt man damit nur dem Rechnung, dass dieses Gesellschaftsformat nicht nur sehr viel älter ist als der Nationalstaat, sondern sich auch einer eigenen, intrinsischen Logik verdankt, nämlich dem Versuch, Mobilität und Vielfalt gezielt als Grundlagen für das Zusammenleben zu nutzen. Diese Einsicht hat nicht nur dazu geführt, sich vom methodologischen bzw. hermeneutischen Nationalismus zu verabschieden, sondern auch dazu, sich bei der Beobachtung, Beschreibung und Analyse der Stadtgesellschaft zunächst ethnographisch auf den urbanen Alltag zu fokussieren und von dort aus die sich zunehmend komplex entwickelnden urbanen Strukturen zu rekonstruieren. Auf diese Weise kann man die Stadtgesellschaft einerseits alltagsweltlich beschreiben und anderseits analysieren, wie sich das Alltagsleben in unterschiedlichen urbanen Kontexten verändert und welche Regeln dabei leitend werden.

Wenn man so verfährt, dann wird schnell klar, dass die Stadtgesellschaft heute damit noch nicht vollständig erfasst ist, weil sie im Rahmen der fort-

3 Aus der Perspektive des Alltagslebens bildet die Stadt - nicht mehr und nicht weniger - den primären Horizont des sinnhaft sozialen Handelns (Max Weber). Alles andere zählt zu „ferner liefen“. Die Stadt konstituiert sich als emergentes lebendes System. 
schreitenden Globalisierung und den damit verbundenen ökonomischen, technischen, medialen und sozialen Entwicklungen einem massiven gesellschaftlichen Wandel unterliegt. Geht man so vor, so erkennt man, dass sich Stadtgesellschaft heute ganz anders als noch zur Zeit der Industrialisierung darstellt. Im Übergang zur Postmoderne hat sich die Stadtgesellschaft zu einem Fußabdruck einer globalisierten Wirklichkeit gewandelt. Auch wenn sie weiter überkommenen grammatischen Strukturen folgt, ist sie genötigt sich ganz anders als bisher aufzustellen. Sie hat es heute mit einer zunehmend diversen und mobilen Bevölkerung zu tun und muss sich auf die Vielen als Viele immer wieder neu einstellen. Und damit entstehen auch ganz andere Herausforderungen, die angefangen beim Verwaltungshandeln bis hin zum Umgang mit sozialen Verwerfungen neu wahrgenommen werden müssen. Das bedeutet auch, dass man lernen muss, ganz anders als bisher mit ihnen umzugehen. Doch gehen wir weiter schrittweise vor:

\section{Was es bedeutet, wenn die Stadtgesellschaft zum Fußabdruck globaler Wirklichkeit wird}

Die Globalisierung prägt über die neuen Medien und Mobilitätsformen sowie die neuen Märkte die Situation in den Stadtgesellschaften. Man kann die Effekte der neuen Medien, Mobilitätsformen und Märkte fast wie einen Fußabdruck überall im urbanen Alltag wahrnehmen. Das gilt zunächst für die alltäglichen Lebensgewohnheiten und für die bauliche Gestaltung der Wohnungen, Gebäude, Straßen, also im Blick auf alles, was wir in der Stadt beobachten und ggf. auch ethnographisch erfassen können.

In Abbildung 1 wird versucht, das entsprechend unter „Globalisierungseffekte“ und unter „Regionaler Fußabdruck“ anzudeuten.

Was man hier beobachten kann, das sind relativ direkte und nicht weiter bedachte, sondern pragmatisch akzeptierte Einwirkungen auf den urbanen Alltag (neue Mobilität, neue Medien, neue Märkte - begleitet von einer zunehmenden Vielfalt, von einer sich ausweitenden Fluktuation der Bevölkerung und von einer zunehmend virtuellen Orientierung) und entsprechend ,anschlussrationale“ Reaktionen auf diese globalen Einwirkungen, mithin ein auf ständige Aktualisierung bedachtes alltägliches Arrangement. Man kann hier von einem ,selbstverständlichen Arrangement“ (taken-for-granted) sprechen. Was sich hinter dieser Selbstverständlichkeit, seine Arrangements immer wieder neu zu inszenieren und darin eine erhebliche Alltagspraxis zu entwickeln verbirgt, das ist das Interesse daran, den Dauerablauf des Alltags aufrecht zu erhalten. Dieses Interesse zwingt dazu, eine ganze Reihe von „Unschärfen“ in Kauf zu nehmen. Tatsächlich gehört dazu sogar eine erhebliche Kunstfertigkeit. Man muss handeln, ohne allzu genau hinzuschauen, 
man muss Widersprüche ertragen und wird Konflikte nicht unbedingt gleich austragen. Man kann dies als eine pragmatisch bestimmte Toleranz bezeichnen. Diese Strategie, die schon Eving Goffman beobachtete und als eine wohlwollend-distanzierte Umgangsweise bezeichnete, ermöglicht in der Regel einen mehr oder weniger tragfähigen modus vivendi und so etwas wie die „Bedingungen der Möglichkeit“ für eine routinierte Bewältigung des Alltagslebens.

Abb. 1: Urban City

\section{- Zur Dynamik einer postmodernen Stadtgesellschaft}

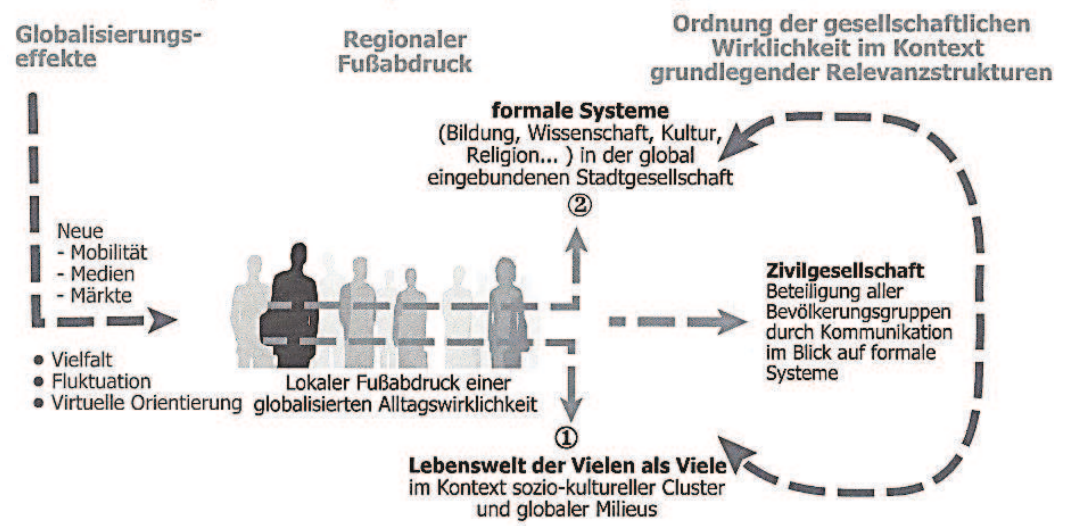

Vorfindliche Gemengelagen in der Form von vordefinierten Situationen, wohlumgrenzten Räumen, als relevant ausgewiesenen Netzwerke werden handelnd umgesetzt. Dazu werden soziale Drehbücher, gerade modische Handlungs- und Deutungsformate aktiviert. Auf diese Weise wird der Dauerablauf des Alltags neu arrangiert. Dabei verändert sich das, was zunächst vorgefunden wurde. Es erfährt Modifikationen. Aus den vordefinierten Situationen werden konkrete Definitionen, aus den wohlumgrenzten Räumen heterotope Räume. Die aktualisierten Drehbücher usw. gehen in neue, in nunmehr hybride Kulturen und Bricolagen ein. Insoweit ist das alltagspragmatisch bestimmte sinnhaft-soziale Handeln ein kreativer, kunstvoller und damit auch toleranter Vorgang. Aber nicht alles, was hier einwirkt, steht zur Disposition.

Vieles entzieht sich dem praktischen Zugriff. Was sich in der Alltagspraxis nicht so einfach verändern lässt, sind alle die Aspekte gesellschaftlicher Wirklichkeit, die als Rahmenbedingungen des Handelns außerhalb der 
Reichweite der Praxis gesellschaftlich verankert sind. Hier kommt es darauf an, wie weit diese Verankerung vom handelnden Subjekt entfernt ist.

a) Vor allem in kleinräumigen Quartieren (Bukow 2010a, S. 101ff.), dann aber auch in urbanen Zwischenräumen (Müller 2010, S. 31f.) und in arrival cities (Saunders 2011) - überall, wo die Rahmenbedingungen vor allem informeller Art sind, wo Umgangsweisen, Ansprüche und Regeln immer wieder kommunikativ zur Disposition gestellt werden, wo Erfahrungssätze, Erzählungen und Traditionsbildung immer wieder neu verfestigt werden müssen - bleibt auch der Handlungskontext variabel, kann die Pragmatik des Alltagshandelns auch die Rahmenbedingungen mit verändern, kann sich aus der Pragmatik so etwas wie eine praktische Vernunft entwickeln.

b) In den großen urbanen Zentren mit einer zentralisierten und homogenisierten Machtstruktur, in den entmischten Cities genauso wie in den Hochhaussiedlungen der Trabantenstädte, aber auch in den kleinbürgerlichen Vorort-Schlafstädten stehen die Orientierungspunkte für das Handeln nicht zur Disposition. Sie sind nicht mehr verhandelbar, nicht mehr erreichbar und werden damit je nach den dominierenden sozialen Mustern und je nachdem, ob es um Insider oder Outsider geht, zu Anknüpfungspunkten für gezielte Inklusion, Segregation oder Exklusion. Sie erzeugen Differenzlinien bzw. Achsen der Ungleichheit, die den Alltag mehr und mehr verbindlich und nachhaltig durchziehen. Sie sind fast immer strukturell und/oder ideologisch verankert und wirken ubiquitär. Das Ergebnis ist eine Relationierung des Alltagshandelns nach Herkunft, Kultur, Klasse vermittels Prekarisierungs-, Diskriminierungs-, Othering-, Ethnisierungs- oder Kriminalisierungsprozessen und anderen Regulations- und Zurechnungspraktiken. Die grundsätzliche Ordnung des Alltags mit ihren intersektionellen Differenzlinien und ihre Verschränkung nach Achsen diverser Versionen von Ungleichheit steht nicht zur Disposition.

Diese Unterscheidung ist wichtig. Sie erklärt zum einen, warum in dichten, sozio-kulturell vermischten, baugeschichtlich wie architektonisch zusammengewürfelten Quartieren das Zusammenleben unproblematischer erscheint und man sich mit dem Quartier identifiziert, obwohl oft eine hohe Mobilität und Diversität herrscht (Bukow 2010b). Und sie erklärt zum anderen, warum in den Hochhaussiedlungen und den sterilen Nachkriegsreihenhäusern die Bevölkerung oft viel homogener ist, zugleich aber ein hohes $\mathrm{Ma}$ an Anonymität und Abgrenzung gegenüber den anderen vorherrscht, was eine Rückzugshaltung und Resignation begünstigt. Nicht in den gemischten und oft wenig ansehnlichen Quartieren, sondern in den homogenen, sterilen Quartieren sieht sich die Bevölkerung sehr viel unmittelbarer und zugleich 
massiver mitunter problematischen und damit als bedrohlich empfundenen Rahmenbedingungen gegenüber. Im ersten Fall entstehen urbane Erfolgsmodelle und im zweiten Fall typische Vorstadtprobleme, für die geradezu sprichwörtlich die französischen Vorstädte stehen (Bukow/Preissing 2010). Dies zeigt, dass eine gute Alltagspraxis zwar so etwas wie eine erfolgreiche Sozialtechnik darstellen mag, aber noch nicht alles ist.

\section{Wie der Fußabdruck globaler Wirklichkeit durch die soziale Grammatik des urbanen Zusammenlebens kontextualisiert und verräumlicht wird}

Halten wir die bisherigen Befunde fest und schauen wir von dort aus, wie das Alltagshandeln nach spezifischen Kontexten geordnet, d.h. verräumlicht wird. Wir haben es in den modernen Stadtgesellschaften jenseits der unmittelbar beobachtbaren und beschreibbaren Alltagspraxis letztlich mit drei deutlich unterschiedlichen Relevanzstrukturen von gesellschaftlicher Wirklichkeit zu tun, die genau diese Kontextualisierung und Verräumlichung hervorrufen. Man kann sie als Verfahren deuten, die je nach der Situation verbindliche Ordnungen generieren. Es sind Relevanz- oder Zurechnungsmuster von je spezifischer Logik, die man als soziale Grammatik des urbanen Zusammenlebens bezeichnen kann (Bukow u.a. 2001, S. 427f.). Dieses Konzept hat sich bei verschiedenen Analysen bewährt, weil es für die Unterschiedlichkeit von Zurechnungen und damit die Unterschiedlichkeit der Regelstruktur innerhalb entsprechender sozialer Situationen sensibel macht.

Der Begriff „Grammatik“ wird in der Stadtanalyse häufiger, aber sehr unterschiedlich verwendet. Im vorliegenden Zusammenhang geht es um ein Konzept, das vom Zusammenspiel dreier deutlich unterschiedlich ausgerichteter Zurechnungs- und Regelungsmodalitäten des Alltagshandelns ausgeht. Es ist eben etwas anderes, ob man sich in einer Wir-Gruppe wie dem Verein (in der Lebenswelt) oder einem formalen System wie einer Verwaltung (in gesellschaftlichen Teilsystemen) oder in einer Debatte, wie sie innerhalb einer Bürgerinitiative stattfinden mag (in der Zivilgesellschaft), bewegt. Es herrschen hier jeweils nicht nur unterschiedliche, sondern zum Teil sogar entgegengesetzte Regeln - vor allem hinsichtlich der Aspekte, die im Zentrum der Stadtgesellschaft stehen, nämlich Mobilität und Vielfalt. In der WirGruppe werden Kontinuität, eine möglichst geringe Mobilität und eine mehr oder weniger verbindliche gemeinsame Identität geschätzt. In der Verwaltung sind Mobilität und Diversität keine zu verhandelnden Eigenschaften, sondern wichtige Themen. In einer Bürgerinitiative sind Mobilität und Diversität wichtige Erfahrungsquellen und damit u.U. alles entscheidende Ressourcen. Die urbane Grammatik ermöglicht auf diese Weise eine auf ein stadtgesell- 
schaftliches Zusammenleben abgestimmte multikontextuelle und funktional dreifach differenzierte Ausrichtung des Handelns. Sie ist es, die den Zusammenhalt der Stadtgesellschaften gewährleistet.

Diese Grammatik des Zusammenlebens macht die Stadtgesellschaften relativ autonom und das keineswegs nur in der noch relativ stark lokal verwurzelten traditionellen Moderne, sondern gerade auch im Umbruch zur Postmoderne. Denn ausgerechnet unter den sich heute massiv verändernden globalen Bedingungen ist diese Grammatik gefragt. Es sieht so aus, also ob sie gerade unter den aktuellen Bedingungen zu einem Erfolgsmodell avanciert ist. Sie hat sich jedenfalls als Gesellschaftsformat überall durchgesetzt und alle anderen Gesellschaftsformen endgültig verdrängt. Anders wäre kaum zu begreifen, dass ganze Stadtregionen wie die Rhein Main Region (5,5 Millionen Einw.), der Rhein Ruhr Raum (11,7 Millionen Einw.) oder Lagos (16 Millionen Einw.) bzw. Mexiko City (21 Millionen Einw.) als einander in dieser „grammatischen Grundstruktur" verwandte Stadtgesellschaften agieren. Insofern gewinnt das urbane Handeln im Kontext einer globalisierten Grammatik des Zusammenlebens deutlich an Stärke und damit auch an strategischer Autonomie. Es ist nur folgerichtig, wenn Stadtgesellschaften diese strategische Autonomie aktiv nutzen, indem sie z.B. Menschenrechte für das urbane Zusammenleben einklagen. ${ }^{4}$

\section{Welche Herausforderungen durch die nach den Regeln der urbanen Grammatik geordnete Verräumlichung der globalen Wirklichkeit entstehen}

Für die vorliegende Fragestellung ist es wichtig, nicht nur genauer zu prüfen, wie sich diese soziale Grammatik des urbanen Zusammenlebens, genauer: wie sich die angedeuteten drei Kontexte des Zusammenlebens (Lebenswelt, System und Zivilgesellschaft) zur Zeit darstellen, sondern vor allem auch, wo vor diesem Hintergrund hier und heute spezielle Herausforderungen zu erkennen sind. Viele, zur Zeit vor allem in der politischen Öffentlichkeit gehandelte Herausforderungen sind im Licht dieser Diskussion mehr als problematisch und lösen häufig sogar kontraproduktive Aktivitäten aus. ${ }^{5}$

4 Die Brisanz dieser Entwicklung zeigt sich erst neuerdings wieder in den Auseinandersetzungen in den arabischen Ländern, wo im Namen der Menschenrechte (einem globalen Verweis) urbane Belange identifiziert und kritisch gegen nationale Strukturen und Rechtssysteme gewendet werden.

5 Hier lässt sich deutlich machen, warum Ludger Pries (2010) ein Konzept „Jenseits von Identität und Integration“ fordert oder Sabine Hess (2009) von „,nointegration?!“ spricht. 
Um deutlich zu machen, welche Herausforderungen durch die nach den Regeln der urbanen Grammatik geordnete Verräumlichung einer längst globalisierten Wirklichkeit entstehen, ist es notwendig, die jeweiligen, situationsspezifischen, eine entsprechend verbindliche Ordnung generierenden Muster herauszuarbeiten, ihre Eigenschaften zu markieren und ihre Implikationen bis hin zur Sozialen Arbeit zu diskutieren. Deshalb soll die Aufmerksamkeit noch einmal explizit auf die drei idealtypisch gemeinten, deutlich unterschiedlich gelagerten Kontexte des Handelns gerichtet werden:

a) Schon der soziographische Blick ermöglicht einen instruktiven Einblick in lebensweltlich definierte Situationen und hier in für Wir-Gruppen typische dichte Interaktion - Situationen innerhalb einer Familie, eines Freundeskreises, einer Bezugsgruppe, eines Stammtisches oder einer Community. Er zeigt aber auch, dass die Lebenswelt der Bevölkerung „diverser“ und „flüchtiger“ wird. Die Bevölkerung wird vielfältiger; es entwickeln sich immer unterschiedlichere familiale und andere wir-gruppenspezfische Lebensstile und zunehmend heterogene, ja hybride Selbstbeschreibungen. Darüber hinaus findet eine Ausdifferenzierung der Lebenswelt zu speziellen Milieus statt, was unter den Bedingungen der modernen Kommunikationsmittel wie Skype zu zunehmend virtuellen Kommunikationsstrukturen (Wir-Cluster) und Diskursgemeinschaften (virtuelle Bezugsgruppen) mit entsprechenden globalen Bezügen führt. Damit ist ein Prozess gemeint, den Ludger Pries mit Transnationalisierung oder „,virtueller Heimat“ beschreibt und als ,grenzüberschreitende Vergesellschaftung" deutet (Pries 2010, S. 169f.). Wichtig ist hier allerdings die Beobachtung, dass diese Entgrenzung sich durchaus mit den dichten Beziehungen, wie sie für lebensweltlich orientiertes Handeln typisch sind, verträgt. Auch über virtuelle Netzte organisierte Interaktionsprozesse können offenbar ,überschaubare“ Orte erschaffen und stabilisieren. Auch virtuelle Interaktion bleibt z.B. über Skype „direkte“ Kommunikation und dient damit genauso der Identifikation und der Identitätsbindung. Man kann es auch daran erkennen, dass die hier gewonnenen Erfahrungen in aller Regel biographisch verankert das ganze Leben „mitgeschleppt“" werden. In der Lebenswelt geht es auf der Basis direkter Interaktion um Vertrauensbildung und um die Anerkennung der jeweiligen individuellen Kompetenzen, Fertigkeiten und Lebensstile im Blick auf alltägliche „,basics“ (hinsichtlich sozialer, sprachlicher, kultureller, religiöser Eigenschaften, der Geschlechtsrolle und des Selbstbildes). 
b) Von hier ist es oft nur ein kleiner Schritt zu einem lokalen Engagement, zu lokalen Publikationen, zu Internetforen, sozialen Netzwerken und Second Life ${ }^{6}$ Damit entstehen aber auch neue Verankerungsmöglichkeiten für zivilgesellschaftliche Aktivitäten und neue Formen gesellschaftlicher Partizipation wie ePartizipation. In diesem Kontext zielt das Handeln überhaupt nicht auf die Verwirklichung und Anerkennung des gesamten Kompetenz- und Persönlichkeitsspektrums des Einzelnen, sondern ganz anders auf die Entwicklung, Ausformulierung und Verallgemeinerung, d.h. Durchsetzung von transindividuellen, kollektiven, der Allgemeinheit zugesprochenen Interessen und Anliegen. Es geht im Kern um die allgemeine, die gesellschaftliche Anerkennung zunächst nur individuell definierter Anliegen durch die Allgemeinheit und damit um Einflussnahme. Hier konkurrieren Vereine, Initiativen, zivilgesellschaftliche Verbände und global agierende Organisationen miteinander um Einfluss und gemeinsam um Einfluss in der politischen Steuerung der Globalgesellschaft. Diese Sphäre gesellschaftlicher Wirklichkeit ist seit ihrer Entstehung im 19. Jahrhundert, ,international“" orientiert. Dies gilt heute mehr denn je. Denn selbst wenn es nur um den Kampf gegen eine lokale Baumaßnahme oder um mehr Rechte für eine Minderheitengruppe geht, wird global mit Ressourcenknappheit und Klimaveränderungen oder Menschenrechten und mehr Chancengleichheit usw. argumentiert. In der Zivilgesellschaft geht es um gleichberechtigte Beteiligung für die Durchsetzung von mehr als nur den Einzelnen betreffenden allgemeinen Anliegen.

c) Last but not least geht es um die Kontextualisierung des Handelns durch formale Strukturen, mithin um die Frage, wie die Alltagspraxis innerhalb der gesellschaftlichen Systeme und Institutionen geordnet wird. Gerade weil die Systeme und Institutionen für die Arbeits- und Lebensfähigkeit der Stadtgesellschaft fundamental sind, kommt es hier darauf an, sie mit der gesellschaftlichen Entwicklung immer wieder neu zu synchronisieren. Sie müssen einerseits für das, was in den anderen beiden Kontexten organisiert wird, sensibel bleiben, also die Vielfalt der Lebenswelten innerhalb der Stadt anerkennen und die in der Zivilgesellschaft entwickelten Impulse ernst nehmen; das Zusammenspiel mit diesen beiden Kontexten wird häufig ignoriert. Sie müssen aber auch im Blick auf ihre eigene Struktur Anschluss halten an die anderen beiden Kontexte genauso wie sie selbst bestimmende globale Entwicklung, d.h. ihre Struktur mit 
allen relevanten externen Trends synchronisieren und sich entsprechend immer wieder neu akkommodieren. Die gesellschaftlichen Systeme und Institutionen sind allerdings von ihrer Intention her nicht nur formal-rational, sondern auch autopoietisch angelegt, d.h. sie funktionieren aufgrund intrinsischer Leitdifferenzen. Wenn sich die Kontextbedingungen heute immer schneller verändern, die Bevölkerung mobiler denn je ist und die Stadt immer diverser erscheint, dann stoßen autopoietisch ausgerichtete Systeme schnell an ihre Grenzen und müssen notfalls von ,außen" neu ausgerichtet werden. Allein um die wichtigsten Komponenten urbaner Existenz durch eine ausreichende Beteiligung an Arbeit, Wohnen, Bildung, Öffentlichkeit, an Mobilität, Kommunikation, Kultur, Gesundheit sowie an der kommunalen Infrastruktur abzusichern, bedarf es einer beständigen Neuanpassung der hier jeweils relevanten Systeme und Institutionen. Gerade die aktuelle Integrationsdebatte belegt, wie schnell Systeme angesichts „unerwartet“ verändernder Rahmenbedingungen dazu ,neigen“, sich an gewohnte und irgendwann institutionalisierte Strukturen zu klammern, statt sich umzustellen, um externen Entwicklungen gerecht zu werden, um z.B. einer veränderten Zusammensetzung der Bevölkerung gerecht zu werden. Die Folge dieser autopoietischen Selbstbeschränkung der Systeme ist hier, dass das Klientel entweder ignoriert oder es zur Anpassung aufgefordert wird - zur Assimilation an die in autopoietischer Selbstbeschränkung immer noch institutionell tradierte, eigentlich längst überholte Wirklichkeit. Aber die Hoffnung auf ein weiter-so-wie-bisher trügt. Angesichts der aktuellen Entwicklungen ist das Problem die ständige Neuausrichtung auf veränderte globalgesellschaftliche Bedingungen im Blick auf ihre innere Struktur, ihre Aufgaben und die Form der Einbeziehung der jeweils aktuellen Bevölkerung als ganzer. Dazu müssen sich die Systeme ganz anders als bisher einstellen, quasi „neu“ erfinden als lebende Systeme.

Ein Blick auf die gewandelten Bedingungen der Stadtgesellschaft verdeutlicht aber auch, wie die fortschreitende Globalisierung bzw. die in diesem Rahmen forcierte technologische Entwicklung und die dadurch ausgelöste zunehmende Mobilität und Diversität die Stadtgesellschaft unter Zugzwang setzt. Was die Reaktion der Stadtgesellschaft betrifft, so wird erkennbar, dass sie zwar im Prinzip über eine bewährte und nach wie vor sehr anpassungsfähige soziale Grammatik verfügt, dass sie aber die vorhandenen Mittel nur zögerlich und wenig durchdacht einsetzt und die Interessen der Vielen als Viele immer wieder ignoriert werden. Die Trägheit der urbanen Systeme und deren mangelhafte Anpassungsbereitschaft erweisen sich immer wieder als Kernprobleme. Die von der Verwaltung und von der lokalen Poli- 
tik bestimmten Systeme sind sicherlich nicht zufällig so „träge“, sondern haben mit der lokalen Privilegienstruktur zu tun, der sich die Kommunen verpflichtet fühlen. Einschlägige Untersuchungen belegen, dass sich Politik und Verwaltung deshalb sehr häufig vereint gegen eine nachhaltige Neueinstellung wehren und einfach auf überkommenen Einstellungen beharren. Die aus solchen ,gestrigen“ Orientierungen resultierenden Fehlleistungen wie Prekarisierung, Segregation und Exklusion werden dann ,gerne“ individualisiert, also die in diesem Zusammenhang entstehenden Verwerfungen und Probleme einfach dem Einzelnen zugerechnet. Und man verwendet schnell Social Engineering-Techniken, um dem Einzelnen die für einen Erhalt des status quo ante mitsamt seiner einstigen Macht- und Privilegienverteilung erforderlichen Rückanpassung im Sinn von „Bring-Leistungen“ aufzubürden. Für die Pädagogik im Bildungssystem, die Sozialpädagogik in den Einrichtungen und die Soziale Arbeit in der Arbeit vor Ort wirkt sich das wie ein „Retro-Sog“, dessen man sich im Grunde nur politisch erwehren kann.

\section{Zur Dynamik des alltäglichen Arrangements}

Die Stadtgesellschaft verfügt über ein differenziertes Potential im Umgang mit Mobilität und Diversität. Es ist aber auch schon deutlich geworden, dass dieses Potential gezielt genutzt und immer wieder zur Neueinstellung des Zusammenlebens aktualisiert werden muss. Die damit markierten Spannungen zwischen einem erfolgreichen Arrangement der Vielen als Viele und dem Unvermögen bzw. der Weigerung, dieses Arrangement zu unterstützen und den daraus erwachsenden Problemen werden noch plastischer, wenn man sich direkt dem pädagogischen Feld, dem Ort sozialer Praxis zuwendet. Der Blick auf eine typische Alltagssituation kann das deutlich machen.

Vom einem völlig selbstverständlichen Aufwachsen im globalisierten Stadtquartier und von einer ganz gewöhnlichen Aneignung von Mobilität und Diversität

Ein Blick darauf, wie Menschen im Stadtquartier völlig selbstverständlich aufwachsen und sich den globalisierten Alltag ganz gewöhnlich aneignen, kann klar machen, wie unspektakulär der Fußabdruck einer globalisierten Wirklichkeit sein kann. Dazu möchte ich auf eine Szene aus dem Alltagsleben von heranwachsenden Kindern in einem typischen Quartier eingehen (Abb. 2: Kinderszene in Bergen). Oben war ja schon mehrfach zur Erläuterung methodischer Fragen auf die Situation von Jugendlichen (vor 
allem am Beispiel der Kalker Ereignisse) Bezug genommen worden. In diesen Überlegungen wurde bereits deutlich, dass die Situation von Jugendlichen im Quartier erst dann prekär wird, wenn man von außen heran tritt und wesentliche Merkmale der Situation verleugnet und aus nationalstaatlicher Perspektive umetikettiert.

Abb.2: Kinderszene in Bergen

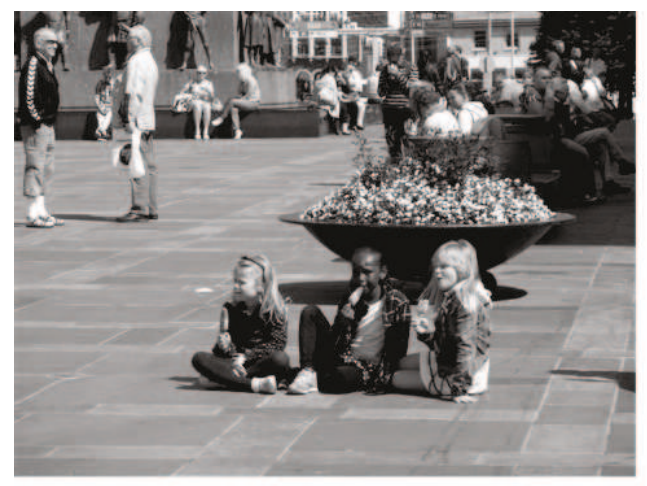

Bei der empirischen Annäherung an die Dynamik des alltäglichen Arrangements ist es hilfreich, sich exemplarisch auf eine typische Szene aus dem Alltagsleben von Kindern, Jugendlichen oder Heranwachsenden zu beziehen. Dabei kommt es darauf an, rekonstruktiv zu verfahren, also aus der Perspektive der Betroffenen zu argumentieren, an deren „empirischen“ Blick anzuknüpfen und erst danach auf Außenansichten einzugehen und die Sicht der im Quartier Aufwachsenden erst ganz zum Schluss mit anderen Sichtweisen bzw. Deutungen zu vergleichen.

Bei dem hier ausgewählten Fallbeispiel geht es darum, wie sich Kinder hier und heute inszenieren, sich gewissermaßen als ,natürliche“ Experten des Alltags betätigen und ihren Alltag so, wie sie das für richtig halten, hervorbringen. Es ist aber auch ein Fall, der zumindest auf den ersten Blick eigentlich gar nicht so leicht so kontextualisiert werden kann, dass man an ihm „Integrationsdefizite“ zu markieren vermag. Das Bild „Kinderszene in Bergen" bildet eine durchaus repräsentative Situation in einem urbanen Quartier ab. Die Situation ist auch in ihrem gesellschaftspolitischen Kontext mit der Situation in einer Stadt wie Köln oder Aachen gut vergleichbar.

Bei der Situation handelt es sich um ein triviales Arrangement zwischen Kindern, die gemeinsam Eis essen. Was im Dauerablauf des Alltags als selbstverständliches Verhalten von Kindern an einem der seltenen Sonnentage in einer Stadt wie Bergen hingenommen wird, das ist für einen aufmerk- 
samen Beobachter ${ }^{7}$ dennoch bemerkenswert. Schon auf den ersten Blick fällt die synchrone Haltung der Kinder auf. Sie signalisiert eine zwischen den Beteiligten übereinstimmende Ausrichtung der Tätigkeit, damit zugleich eine prinzipielle Übereinstimmung in der Handhabung der Situation, also im Situationsformat und damit schließlich auch in der Situationsdefinition. Die Situationsdefinition ,gemeinsam Eis-Essen“ wird zum Orientierungspunkt, systemtheoretisch formuliert zur Leitdifferenz der Situation und ermöglicht die Aktualisierung entsprechender Handlungs- und Verhaltensregeln.

Die Situation erhält eine besondere Pointe durch das Auf-dem-BodenSitzen. Es ist eine spezielle Handlungsweise, die einer besonderen Konstellation, nämlich Sonnenschein in Europas an sich regenreichster Stadt, geschuldet ist. Es ist durchaus bemerkenswert, wenn diese drei Kinder hier auf dem Straßenboden sitzen, was allerseits gelassen hingenommen $\mathrm{zu}$ werden scheint. Es gibt offenbar zwischen den Kindern und den Passanten eine stillschweigende Übereinstimmung in dem aktuellen Handlungsformat Auf-demBoden-Sitzen als einer legitimen Variation des Aufenthalts in einem öffentlichen Raum angesichts des extrem seltenen Sonnenwetters. Alle verfügen in dieser Situation in Bezug auf den Umgang mit der Situation über einen vergleichbaren Wissensstand, obwohl alle beteiligten Personen eben auch einschließlich der wie selbstverständlich vorbei kommenden Passanten ebenso offensichtlich im Blick auf Alter, soziale Struktur und Herkunft deutlich unterschiedlich orientiert sein dürften.

Zunächst einmal ist diese Szene sicherlich ein Beispiel dafür, wie in einer konkreten Alltagssituation für die Teilnehmer viele ansonsten u.U. (für den Beobachter als Unbeteiligten mitunter sofort ins Auge fallenden) signifikante Unterschiede konstitutiv belanglos bleiben. Sodann ist sie ein Beispiel dafür, wie selbstverständlich dabei Erfahrungen mit einem Alltag gemacht und angeeignet werden, die aus der Sicht des Beobachters hoch komplex und heterogen, ja widersprüchlich erscheinen mögen. So ist einem kulturgeschichtlich informierten Beobachter natürlich klar, dass sich die gesamte Thematik - es handelt sich um den Konsum von Eis-am-Stiel - der Globalisierung, genauer der letzten Globalisierungswelle verdankt und „eigentlich“ überhaupt nicht zum lokalen Lebensgefühl passt. Das Situationsformat ist noch nicht alt und wäre noch vor zwei Generation als hybrid wahrgenommen worden (Bukow 2010b, S. 207ff.). Heute scheint das Format längst alltäglich, irgendwie trivial zu sein und ist fast schon Brauchtum.

7 Einer der häufigsten Fehler in der Sozialforschung besteht darin, bei einer Beobachtung die einem als Externen zwangsläufig unvertraute, dem Handeln jedoch inhärente sinnhaftsoziale Logik zu ignorieren und nach „zufälligen“, d.h. nach situationsfremden, der eigene Rolle innerhalb des Alltags geschuldeten Vorstellungen und Kriterien vorzugehen. 
Wie aus trivialen Alltagsaktivitäten in einem globalisierten Alltag wie selbstverständlich hybride Identitäten entstehen

Betrachtet man die Situation sozialisationstheoretisch, so kann man den Fall dafür anführen, wie an einer konkreten Thematik unter Absehen von allen „sonstigen“ Unterschiedlichkeiten der beteiligten Personen eine Situationsdefinition ausgehandelt und entsprechende Regeln aktiviert werden, um zu einer gemeinsamen Handlung zu kommen und ein gemeinsames Handlungsziel zu erreichen. Das dabei kreativ abgearbeitete soziale Format geht in den Wissensbestand der Beteiligten ein und wird, positiv erfahren, zu einem Bestandteil der individuellen Entwicklung. Insofern handelt es sich hier einerseits um einen durch sozialisatorische Interaktion erzeugten Erfahrungsgewinn und anderseits zugleich auch um einen von den Beteiligten erzeugten Beitrag zur Fortschreibung von urbaner gesellschaftlicher Wirklichkeit.

Berücksichtigt man die Tatsache ein, dass die Thematik in dieser Form erst zwei Generationen alt ist und sich somit nicht nur die Handelnden, sondern auch deren Praxis der Globalisierung verdanken, so bietet die Szene zugleich eine eindrucksvolle Antwort auf die Frage, wie aus einer im Prinzip hybriden Welt mit der Zeit eine hybrid basierte Identität entsteht. Dies gilt in zweierlei Hinsicht: Zum einen lernt man, im Alltag stets situationsspezifische Maßstäbe anzulegen und dabei die den unterschiedlichen Situationen jeweils typisch verschiedenen Regeln zu verwenden. Man erwirbt die Fähigkeit, das je nach der Situation passende alltagskulturelle setting anzuwenden und sich die entsprechenden Vorstellungen, so etwas wie eine hybrid cognitive map, $\mathrm{zu}$ eigen $\mathrm{zu}$ machen. Und zum anderen lernt man damit automatisch auch, dass, da die Alltagswelt nicht geschlossen und eindeutig, sondern nur in typisch unterschiedlichen Situationen existiert, es notwendig ist, sich die Dinge selbst zusammen zu reimen. Die hybrid cognitive map nötigt zu einer individuellen Bricolage der Vorstellungen von sich in der Welt. Es mag für den Beobachter erstaunlich sein, wie virtuos das bereits Kinder und Jugendliche bewältigen. Für die Beteiligten ist dies längst selbstverständlich, eine conditio sine qua non.

Der skizzierte wie selbstverständliche, gleichwohl aber virtuose Umgang mit Mobilität und Diversität belegt, wie hilfreich die Grammatik des Zusammenlebens ist und wie einfach sie eine Stadtgesellschaft zusammen zu halten vermag, wenn sogar schon Kinder in der Lage sind, diese Grammatik so fraglos wie erfolgreich anzuwenden. Diese Szene mit den drei Kindern ist einerseits völlig trivial, anderseits extrem informativ, weil an ihr sichtbar wird, wie selbstverständlich man sich in einem Quartier zu bewegen vermag, selbst wenn es längst zum Fußabdruck einer globalisierten Wirklichkeit geworden ist. Und sie lässt erkennen, warum sich Kinder, Jugendliche und genauso Heranwachsende in ihrem Quartier in der Regel „trotz allem“ wohl 
fühlen und sich mit dem Ort ihres Aufwachsens identifizieren, selbst wenn sie noch nicht einmal im Quartier geboren sind.

\section{Was aus dem Alltagsarrangement für die Logik einer sozialen Intervention folgt}

Betrachtet man die skizzierte alltägliche Dynamik, so kann man von einer alltagseingebetteten inhärenten sozialen $\operatorname{Logik}^{8}$ des alläglichen Arrangements und damit auch des Aufwachsens ausgehen. Aufwachsen bedeutet, das Handeln und Deuten aus der individuellen Praxis im Quartier, aus alltäglicher Interaktion mit anderen heraus für sich immer wieder neu zu arrangieren und auf diese Weise Schritt für Schritt ein Bild von sich in der Welt zu entwickeln. Aufwachsen bedeutet dann, aus dem Hier und Heute heraus unter Einbeziehung immer neuer Praxiserfahrungen „reflexiv“ Erfahrungen zu gewinnen und in das eigene Welt- wie Selbstbild einzuarbeiten.

Wenn im Verlauf dieses Prozesses Probleme auftreten, dann dürften sie nach diesem Ansatz in der Regel darauf beruhen, dass wichtige „Bausteine“ für das alltägliche Arrangement und die daraus resultierende Entwicklung eines Welt- wie Selbstbildes fehlen: fehlende Gelegenheiten zur Interaktion, fehlende ökonomische, soziale bzw. kulturelle Ressourcen, fehlende Unterstützung innerhalb einer zunehmend komplexeren Alltagswelt. Für die Logik einer sozialen Intervention folgt daraus, dass sie Maßnahmen entwerfen muss, die zur Unterstützung jener intrinsischen Logik des Arrangements wichtig sind, z.B. bei fehlenden Bausteinen unterstützend wirken. Soziale Intervention meinte danach eine kritische Begleitung eines gemäß ,,inhärenter Logik" situierten sozialen Handelns (vgl. Bommes/Scherr 2012, S. 220). Dies kann ihr freilich nur gelingen, wenn sie - um weiter am Beispiel entlang zu argumentieren - von der Lebenswirklichkeit der Kinder und Jugendlichen und ihrer im Prinzip virtuosen Bewältigung der Alltagspraxis ausgeht, d.h. sie grundsätzlich anerkennt und unterstützt. Dazu muss sie unabhängig von externen Interessen agieren, darf sich weder von Behörden und Institutionen in Dienst nehmen lassen, noch darf sie eine Umetikettierung der Alltagspraxis im Dienst eines hermeneutischen Nationalismus akzeptieren, geschweige denn sie sogar selbst mit vornehmen.

Was passiert, wenn die Situation umetikettiert wird? Diese Frage ist keineswegs rhetorisch, sondern stellt sich beispielsweise, wenn man, wie das aus vielen Fällen vertraut ist, den Kindern oder Jugendlichen ihre alltagslogisch

Mit dem Begriff soziale Logik soll an die von Erving Goffman skizzierte Dynamik themenzentrierter, wohlsituierter Interaktion angeschlossen werden, wie er sie in seiner Arbeit über „Spaß am Spiel“ entwickelt hat (Goffman 1973). 
gewonnenen Erfahrungen abspricht und sie, wie es bei Allochthonen fast täglich geschieht, mit der Frage nach deren Geburtsort, bzw. dem der Eltern oder Großeltern zu Fremden stilisiert. Notfalls bedient man sich auch des Kleidungsstils oder der Hautfarbe, um dieses othering $\mathrm{zu}$ erreichen. Und diese Praxis beschränkt sich nicht auf öffentliche Plätze und dort aktive Ordnungskräfte. Tagtäglich werden ,geeignete“ Kinder und Jugendliche diesem othering ausgesetzt, werden deren Ansprüche, Wünsche und Erwartungen destruiert und werden Sozialisationsdefizite postuliert. Kinder, Jugendliche und Heranwachsende werden besonders "gerne“ benutzt, um das Aufeinander-Treffen von Effekten zunehmender Mobilität und Diversität und von Effekten nationalstaatlicher Selbstinterpretation zu beschwören und zu dramatisieren. Wenn soziale Intervention sich hier auf die Seite mobilitäts- und diversitätsfeindlicher „Retro-Einstellungen“ schlägt, übernimmt sie innerhalb der nationalistischen Dramaturgie die Rolle eines Regisseurs. Da die Betroffenen jedoch die ihnen vertraute Quartier-,,Gemengelage“ als eine selbstverständliche und vertraute gesellschaftliche Wirklichkeitskonstruktion erleben, erfahren und verinnerlichen und sich dem entsprechend platzieren, erzeugt diese erfahrungsfremde nationalistische Zumutung fast automatisch Bedrohungsgefühle und Exklusionsängste.

Ein alltägliches Beispiel für diese Umetikettierung ${ }^{9}$ ist die Frage an jemanden, dessen Verhalten, Kleidung und/oder Hautfarbe vom „biodeutschen“ Selbstbild abweicht, wo er her kommt. Das folgende Zitat von Ekrem Senol kann die entsprechenden Implikationen plastisch machen:

„Ja, ich komme ursprünglich aus der Türkei, bin aber hier geboren und aufgewachsen. Dies ist immer öfter die Antwort auf eine entsprechende Frage beim gesellschaftlichen oder beruflichen Kennenlernen, so als wolle man sich abgrenzen vom übrigen Pack. Wer aber sind die Übrigen? Die eigenen Eltern? Der Zusatz, dass man hier geboren und aufgewachsen ist, soll dem Gegenüber klarmachen, dass man dazugehört. Dieser Abgrenzungsversuch signalisiert aber noch viel mehr. Darin schwingt unhörbar Angst mit, Angst, nicht gemocht $\mathrm{zu}$ werden und auf Ablehnung zu stoßen. Angst, in dieselbe Schublade gesteckt zu werden wie die Frau mit dem bunten Kopftuch, die stets von hinten zu sehen ist und die Aldi Tüte in der einen, das Kind in der anderen Hand hält und in den Medien üblicherweise für Nachrichten über Parallelgesellschaften und Hartz IV Empfänger herhalten muss. Gleichzeitig offenbart ein solcher Abgrenzungsversuch, dass man sich von den Integrations- und Islamdebatten in höchstem Maße persönlich angesprochen fühlt. Menschen mit einem gesunden Selbstvertrauen und Selbstwertgefühl zeichnen sich aber gerade dadurch aus, dass sie kein übertriebenes Bedürfnis haben, von allen anerkannt und gemocht zu werden. Sie besitzen die Selbstsicherheit, mit Ablehnung umzugehen, weil sie

9 Es ist ein „gesellschaftliches Manöver mit erheblichen Konsequenzen für die Betroffenen“ zitiert Adele Clarke ganz im vorliegenden Sinn Michel Foucaults (dazu Clarke 2012, S. 97). 
eine Ablehnung nicht persönlich nehmen. Ihr Selbstwertgefühl wird durch eine Ablehnung nicht verletzt oder herabgesetzt" (Senol 2011, S. 54).

Die durch das othering überhaupt erst erzeugten Bedrohungsgefühle und Ängste rufen dann in der Art einer self-fullfilling-prophecy genau das hervor, was später den Einsatz einer ganzen Integrationsindustrie legitimiert. Je intensiver die Dramaturgie des othering betrieben wird, desto plausibler muss es gesellschaftlichen Institutionen erscheinen, womit sie sich schon immer konfrontiert glauben, nämlich mit Integrationsproblemen. Umso mehr sind sie davon überzeugt, sie müssten diese Menschen über vorschulische Erziehung überhaupt erst sprachfähig machen („Null Sprachlichkeit“ überwinden), über besondere Bildungsmaßnahmen (Überweisung in Förderschulen) schulfähig machen und die Familien insgesamt über interkulturelle und interreligiöse Dialoge usw. für das Ankommen in der Gesellschaft motivieren, für das Leben im Quartier überhaupt erst ertüchtigen.

Für den pädagogisch orientierten Beobachter, der sich an der Alltagsrealität des Heranwachsens im Quartier orientiert, ist der hier und heute gelebte Alltag zunächst einmal unproblematisch und bleibt es auch, solange er es nicht mit Kindern zu tun hat, die für dieses Eis kein Geld haben oder mit Zuschauern, die das Verhalten der Kinder unangemessen finden, weil es nicht ihrem Weltbild entspricht. In beiden Fällen ergibt sich die Notwendigkeit, die Situation pädagogisch „zu begleiten“, was allerdings voraussetzt, dass das Format als ein mögliches und fraglos gegebenes Format nicht nur akzeptiert, sondern auch als ein Ausdruck individueller Alltagsgestaltung positiv eingeschätzt wird. Der pädagogische Beobachter benötigt demnach für eine erfolgreiche Intervention nicht nur soziale, sondern auch politische und alltagskulturelle Kenntnisse und er muss einschätzen können, ob das Format zur Entwicklung positiv beiträgt.

\section{Zu Problemen in den „wohlgeordneten“ Kontexten einer Stadtgesellschaft}

In der bisherigen Diskussion habe ich mich darauf konzentriert, eine zumeist undramatische, diversitäts- und mobilitätsgeprägte Alltagspraxis zu skizzieren - aber auch zu prüfen, was passiert, wenn jemand diese Praxis von der Warte eines überholten Gesellschaftsbildes aus nationalistisch dramatisiert. Das bislang gezeichnete Bild bleibt allerdings grobmaschig. Erst wenn man die Alltagspraxis einerseits und die sehr unterschiedlichen nationalistischen Dramatisierungsversuche anderseits genauer diskutiert, d.h. diese beiden Verfahren der Herstellung von gesellschaftlicher Wirklichkeit in den 
oben bereits skizzierten gesellschaftlichen Kontexten Lebenswelt, Zivilgesellschaft und gesellschaftliche Systeme genauer analysiert, kann man präzise Aussagen zu einzelnen Handlungsfeldern und ihren Implikationen machen.

Auch wenn es hier nur um Anfragen an die Praxis der Sozialen Arbeit in einer von Mobilität und Vielfalt geprägten Situation geht, würde dies den Rahmen bei weitem sprengen. Zum einen sind pädagogische Expert/innen in allen drei Kontexten tätig und zum anderen treten sie oft nicht allein auf, sondern sind im Rahmen von Initiativen, Vereinen, Wohlfahrtsorganisationen, Behörden und staatlichen Bildungseinrichtungen tätig. Sie agieren also oft im Verbund und müssen in diesen Fällen jeweils im Blick auf den Verbund, in dem sie auftreten, gesehen werden. Wenn ich mich in der abschließenden Skizze (vgl. Abb. 3) auf soziale Intervention beschränke, so dient das einer nicht ganz unproblematischen Komplexitätsreduktion. Was aber auch so klar wird, ist, dass es vor allem auf die Korrespondenz zwischen der jeweiligen speziellen sozialen Logik einer Alltagspraxis innerhalb von Lebenswelt, Zivilgesellschaft und gesellschaftlichen Systemen einerseits und den Intentionen typischer Formen sozialer Intervention anderseits ankommt.

Im Folgenden werden zunächst die Kontexte Lebenswelt und Zivilgesellschaft angesprochen. Dabei sollen aber nur einige kursorische Hinweise gegeben werden. Die Aufmerksamkeit gilt abschließend vor allem den gesellschaftlichen Systemen und Institutionen, weil sie für die Ordnung des $\mathrm{Zu}-$ sammenlebens letzten Endes entscheidend sind. Hier müssten eigentlich zumindest zwei gegenwärtig alles bestimmende Themen angesprochen werden, nämlich die Bildungsdebatte und die Diskussion über die jüngste Einwanderung aus Südosteuropa. Die Debatte über die Zuwanderung aus Südosteuropa ist freilich besonders gut geeignet, um die Probleme der sozialen Intervention deutlich zu machen, weil anders als in dem Bildungssystem die Rolle der Sozialen Arbeit hier von ganz zentraler Bedeutung ist. 
Abb. 3: Zum Verhältnis von sozialem Handeln und sozialer Intervention

\begin{tabular}{|c|c|c|c|c|c|}
\hline & Praxisfelder & soziale Logik & $\begin{array}{l}\text { soziale } \\
\text { Inter- } \\
\text { vention }\end{array}$ & Intention & $\begin{array}{l}\text { Interventions- } \\
\text { kontext }\end{array}$ \\
\hline Lebenswelt & $\begin{array}{l}\text { Wir-Gruppe, } \\
\text { Stammtisch, } \\
\text { Bezugsgruppe }\end{array}$ & $\begin{array}{l}\text { wertorientierte } \\
\text { Gemeinschafts- } \\
\text { pflege, Einbettung }\end{array}$ & \multirow{3}{*}{\multicolumn{3}{|c|}{$\begin{array}{l}\text { Verhält sich die soziale Intervention } \\
\text { symmetrisch komplementär oder schließt sie } \\
\text { sich z.B. dem hermeneutisch-nationa- } \\
\text { listischen Blick einer Kommunalverwaltung } \\
\text { oder einer Behörde an? } \\
\text { Verfolgt sie ein inklusives Konzept für die } \\
\text { Vielen als Viele oder versucht sie die } \\
\text { Auswirkungen von Mobilität und Diversität } \\
\text { z.B. durch kulturalistische Integrationskon- } \\
\text { zepte an eine „Nationalkultur“ zu assimilie- } \\
\text { ren? }\end{array}$}} \\
\hline $\begin{array}{l}\text { Zivilgesell- } \\
\text { schaft }\end{array}$ & $\begin{array}{l}\text { Initiative, Verein, } \\
\text { pol. Bündnisse }\end{array}$ & $\begin{array}{l}\text { deliberativer Kampf } \\
\text { um Anerkennung }\end{array}$ & & & \\
\hline $\begin{array}{l}\text { gesell- } \\
\text { schaftliche } \\
\text { Systeme }\end{array}$ & $\begin{array}{l}\text { Arbeitsmarkt, } \\
\text { Bildung, } \\
\text { Gesundheit, } \\
\text { Kirchen usw. }\end{array}$ & $\begin{array}{l}\text { thematische Inklu- } \\
\text { sion auf der Basis } \\
\text { von Fairness }\end{array}$ & & & \\
\hline
\end{tabular}

Warum soziale Intervention in den völlig unterschiedlich ausgerichteten Kontexten Lebenswelt und Zivilgesellschaft mitunter konträren Intentionen folgen muss

Zunächst möchte ich einige eher kursorische Bemerkungen dazu machen, warum sich soziale Intervention in Lebenswelt und Zivilgesellschaft nicht nur in völlig unterschiedlich ausgerichteten Kontexten bewegt, sondern auch fast schon konträr ausgerichtet werden muss.

a) In der Lebenswelt geht es um face-to-face Interaktion von Wir-Gruppen, mithin um dichte Beziehungen, wechselseitige Vertrauensbildung und um die Anerkennung der jeweiligen individuellen Kompetenzen, Fertigkeiten und Lebensstile der basics hinsichtlich sozialer, sprachlicher, kultureller und religiöser Eigenschaften, der Geschlechtsrolle und des Selbstbildes. Die hierbei erkennbare soziale Logik besteht darin, sich im Verlauf der individuellen Entwicklung in diesem Kontext reziprok, aktiv und nachhaltig einzurichten. Und wenn man wie gezeigt, auf Kinder und Jugendliche blickt, so sieht man schnell, wie sie dabei alles an Diversität und Mobilität, alles was um sie herum Wirklichkeit wird, wie selbstverständlich einbeziehen. Um es bildlich zu formulieren: hier geht es um ein auf alltäglicher Interaktion basierendes Jagen und Sammeln in subjekti- 
ver Absicht. Wenn hier soziale Intervention gefragt ist, dann nicht top down mit einer von oben bzw. außen implementierten Zielvorgabe („Werte des Abendlandes“, „Leitkultur“ usw.), sondern bottom up und hier im Sinn von kritischer Solidarität mit dem Prozess des „Jagens und Sammelns“. Es ist klar, dass in einem derartigen Alltag gewonnen und ggf. durch soziale Intervention verstärkte Resultate mindestens so hybrid ausfallen wie der Alltag selbst. Dies hat den Vorteil, dass auf diese Weise ein Spielraum für ein Höchstmaß an kollektiv fundierter Individualität entsteht, allerdings auch den Nachteil, dass es kaum mit dem immer noch gepflegten monolingualen bzw. monokulturellen Habitus zusammen passt und dass damit in einer Gesellschaft, die einem überkommenen Kulturmodell aufsitzt, massive Anerkennungsprobleme programmiert sind. Und wenn die hier involvierte Bevölkerungsgruppe ohnehin schon als „Ausländer“ unter besonderer Beobachtung steht, stößt eine auf diesen Menschen orientierte soziale Intervention sehr schnell auf politische Barrieren.

b) In der Zivilgesellschaft geht es wie oben skizziert um die Formulierung von Anliegen gegenüber der Öffentlichkeit und damit um die Beteiligung auf gleicher Augenhöhe zur Durchsetzung von mehr als nur den Einzelnen betreffenden allgemeinen Positionen. Klar ist, dass der Einzelne hier eine völlig andere Rolle einnimmt und damit die soziale Logik ganz anders ausgerichtet ist. Hier geht es gerade nicht um das, was die Lebenswelt im Kern ausmacht, nicht um den Ausbau der Gesellschaft zu einer Groß-Wir-Gruppe, sozusagen einer Großfamilie mit einem Patriarchen an der Spitze, sondern um klug durchdachte und kenntnisreich entwickelte Vorschläge für den Umgang mit gesellschaftlichen Herausforderungen von allgemeinem Interesse. Wenn hier soziale Intervention gefragt ist, dann nicht im Rahmen der individuellen Motivsuche oder der Persönlichkeit, der religiösen Einstellung oder des Charakters der Aktivist/innen, sondern in einem eher technischen Sinn bei der Beschaffung, Gestaltung, Abstimmung und Durchsetzung und dem Publik-Machen von Anliegen. Und so wie die Aktivist/innen nur in ihren speziellen Anliegen übereinstimmen müssen, während alles andere konstitutiv belanglos ist, so wird sich die soziale Intervention auf die Unterstützung der Anliegen zu konzentrieren haben. Auch hier ist klar, dass die unterschiedlichen Motive der zivilgesellschaftlichen Akteure durchaus Probleme machen können - aber dies vor allem dann, wenn man glaubt, ein Anliegen könne nur dann akzeptiert werden, wenn die richtige Motivation oder die richtige Persönlichkeit dahinter steht. Gerade in sozial, kulturell und religiös diversen Gesellschaften funktioniert eine solche 
normative Erwartung nicht. Sie sorgt nur für Zündstoff und provoziert Rassismen und Ausgrenzung. In all diesen Fällen kommen auf soziale Intervention schnell extreme Herausforderungen zu, nämlich der Kampf um gleiche Augenhöhe für die Vielen als Viele.

Wenn die soziale Logik in den beiden Kontexten unterschiedlich funktioniert, so muss sich auch die soziale Intervention entsprechend unterschiedlich ausrichten. Man steht damit vor einem doppelten Problem, nämlich einerseits davor, die soziale Logik der jeweiligen Situation sozial adäquat „lesen“ zu lernen, und anderseits davor, gegenüber Dritten diese soziale Logik zu verteidigen, die ja eigentlich nur der Grammatik des urbanen Zusammenlebens geschuldet ist und die gerade deshalb dann oft nicht akzeptiert wird, wenn sie von den Vielen als Viele mit ihren Mobilitäts- und Diversitätserfahrungen in Anspruch genommen wird. Eigentlich ist es ganz einfach: Im Blick auf die Lebenswelt geht es um eine Stärkung der Bindung innerhalb der Wir-Gruppe unter Ausklammerung von beruflichen, politischen, religiösen und anderen dem gesellschaftlichen Statuserhalt dienenden Praktiken, während es in der Zivilgesellschaft ganz im Gegenteil um den Austausch von Argumenten und damit um eine gezielte Platzierung dieser Argumente in zentralen Segmenten der Öffentlichkeit unter Absehung von der jeweiligen individuellen Einstellung oder persönlichen Sympathie geht.

\section{Warum soziale Intervention in ,wohlgeordneten “ institutionellen Kontexten oft auf erhebliche Schwierigkeiten stößt}

Das Handeln ist in systemischen Kontexten, in den Behörden, in der Politik, in den Bildungseinrichtungen, im Gesundheitssystem wie oben skizziert mit einer autopoietischen, sich intern regulierenden Selbstaussteuerung konfrontiert. Dies ist schon immer problematisch gewesen und war einer der Gründe für die Entwicklung einer kritischen Öffentlichkeit bzw. Zivilgesellschaft. Noch problematischer wird es heute, weil die ohnehin trägen Systeme mit sich schnell wandelnden gesellschaftlichen Entwicklungen konfrontiert sind. Sie sind gezwungen, sich auf zunehmend komplexere, d.h. flüchtigere und diversere Rahmenbedingungen einzustellen. Das bedeutet, sie müssen ihre innere Struktur, ihre Aufgabenstellungen und den Umgang mit den Menschen immer wieder radikal überprüfen, sich wie oben angemerkt quasi als „lebende Systeme“ immer wieder „neu“ erfinden.

Wir haben es hier mit einer auch für soziale Intervention ungewöhnlich problematischen Situation zu tun. Sie zwingt dazu, anders als in den anderen beiden Kontexten etwas ausführlicher zu werden - selbst wenn man nur exemplarisch deutlich machen kann, mit welchen Herausforderungen es 
soziale Intervention hier zu tun hat. Zwei Beispiele bieten sich dafür besonders an, zum einen die Situation im Bildungssystem, wo es zunehmend darum geht von einem integrativen zu einem inklusiven Konzept zu kommen, und zum anderen die Situation in einer Kommunalverwaltung, wo man sich nach vierzig Jahren Einwanderung angesichts einer immer noch anhaltenden, ja neuerlich sogar wieder verstärkten Einwanderung, dieses Mal einer „Zuwanderung“ aus Südosteuropa, genötigt sieht, Einwanderung endlich als normal zu akzeptieren. In beiden Fällen liegen die Herausforderungen für eine angemessene Konzeptionalisierung von sozialer Intervention auf der Hand. Allerdings dürfte die Einwanderungsthematik als solche im Augenblick wieder einmal besonders spannend sein. Und anders als im Bildungssystem spielt hier die Soziale Arbeit eine ganz zentrale Rolle.

Schaut man sich die Strategien an, mit denen die meisten hiesigen Kommunen auf die jüngste Einwanderung, die aus auf Südosteuropa, reagieren ${ }^{10}$, so erkennt man schnell, wie zäh sich trotz vieler von „Willkommenskultur“" vollen Integrationskonzepte die überkommenen einwanderungsfeindlichen Einstellungen erhalten haben und dass man weiter wie gewohnt negativ reagiert. Die Praxis der Kommunen bei der Bewältigung der Einwanderung durch die „Generation Gastarbeiter" vor gut vierzig Jahren hat sich gehalten und ist meist immer noch die Praxis bei dem Umgang mit neuen Bevölkerungsgruppen - allerdings mit dem Unterschied, dass man damals vom Gast sprach, der wieder zu gehen hat, und heute vom Zuwanderer, der sich als ein Hinzukommender ein- und unterzuordnen hat.

Nach wie vor ist man nicht bereit, die Einwanderung in der ihr gewissermaßen eigensinnigen Dynamik wirklich voll zu realisieren, also den betreffenden Menschen das Recht auf ein Zu-bleiben-wie-sie-sind zu konzedieren. Die kommunale Praxis ist träge und neigt offenbar besonders in kritischen Augenblicken dazu, rückwärts auszuweichen, obgleich die Einwanderung längst unumkehrbar ist, sich Dank der aktuellen Globalisierung sogar noch immer weiter ausweitet und oft schon in Fluktuation mündet und eigentlich gar nichts übrig bleibt, als die zunehmende Mobilität und Diversität im Rahmen der Stadtentwicklung aufzugreifen und systematisch zu verarbeiten. Die jüngste Einwanderung hat die Debatte sogar wieder zurück geworfen. Plötzlich spricht man wieder betont negativ von „Armutsflüchtlingen", als ob die Generation Gastarbeiter oder die Übersiedler aus den ehemaligen GUS-Staaten nicht auch arm gekommen seien. Im Grunde geht es darum, dass mancher unterstellt, diese Menschen wären „,von Natur aus“ arm, hätten die Diskriminierung schon im Herkunftsland „verdient“ gehabt und

10 Ich beziehe mich im Folgenden auf Erfahrungen im Rahmen einer wissenschaftlichen Begleitung zweier Kommunen aus Nordrhein-Westfalen. Die Arbeit ist noch nicht abgeschlossen. Die Veröffentlichung der Ergebnisse ist Mitte 2013 zu erwarten. 
würde ihre Armut wie eine Krankheit mit importieren. Ihr Ziel sei nur, mit Hilfe der EU ihr „Lotterleben“ weiter zu optimieren. Viele Behördenvertreter sehen sich dieser Einwanderung deshalb irgendwie „hilflos ausgeliefert" und genötigt als „letzte“ Instanz (nachdem nämlich das Herkunftsland und die EU versagt haben) einzugreifen, die Notbremse zu ziehen, nachdem alles wie nicht anders zu erwarten "schief“ gelaufen sei. Man gestaltet nicht, man interveniert am Ende - man lässt die Einwanderer in problematischen „Zwischenräumen" auflaufen; man duldet, dass sie auf schlechten Wohnraum angewiesen sind, in ausbeuterische Arbeitsverhältnisse geraten. So besehen bleiben dann nur noch ordnungspolitische Maßnahmen. Damit wird ein „sich selbst bestätigender Teufelskreis“" installiert, der im Verlauf der Zeit immer dramatischere Züge annimmt. Man warnt vor Neigungen zur Prostitution, die freilich ausschließlich von den hiesigen Freiern beansprucht wird und plädiert schließlich ganz wie zur Zeit des ersten Ölschocks für Rückkehrmaßnahmen. So bleibt es dann eben doch wieder bei den Reaktionen wie gestern. Sie sind schwer zu stoppen und es fällt den Verwaltungen sehr schwer, sich dieser falschen, offenbar verwaltungssystemimmanenten Dynamik zumal in Zeiten knapper Kassen zu entziehen, zumal die Medien im aktuellen Fall diese falsche Dynamik noch durch eine skandalisierende und kriminalisierende Berichterstattung anheizen und den Einwanderern in kulturrassistischer Manier auch noch mit einschlägigen Reportagen untermauert, unterstellen, sie seien quasi von Natur aus unfähig, ihre Kinder angemessen zu erziehen, einem normalen Erwerb nachzugehen, ein Minimum an Hygiene und überhaupt eine mitteleuropäische Wohnkultur zu entwickeln.

Immer wieder bricht die gewohnte Logik durch, die top down zur Sache kommt. Es fehlt noch immer an einer ausreichenden Sensibilität gegenüber der empirischen Realität - der Realität, die wie beschrieben als lokaler Fußabdruck einer längst globalisierten Alltagswirklichkeit zu deuten ist. Die gewohnte Logik verweigert sich dieser Sensibilität. Und je dramatischer man die Wirklichkeit verfehlt, umso intensiver sucht man nach social-engeneering -Modellen, die vielleicht doch noch etwas erreichen. Um die gebotene Sensibilität zu entwickeln, bedarf es eines Perspektivenwechsels weg vom systemimmanenten Blick hin zu einem kritischen Blick auf die Entwicklungsdynamik von Einwanderung in eine Stadtgesellschaft.

So lange die - die systemtheoretisch gesprochen - Leitdifferenz der Kommunalverwaltung noch nicht grundsätzlich neu bestimmt und das Verwaltungssystem auf das Konzept eines lebenden Systems umgestellt ist, solange bleibt die Verwaltungslogik ein Problem und ist kaum dazu geeignet, sich an sie im Rahmen sozialer Intervention anzukoppeln. Die hier gefragte Logik für eine sozial adäquate soziale Intervention erschließt sich eben nicht aus einer der internen Struktur der Verwaltungen immanenten Handlungslo- 
gik, sondern muss von Grund auf neu und eigenständig Schritt für Schritt ausgehandelt werden.

a) Der erste Schritt könnte darin bestehen, die gesellschaftliche Sinnhaftigkeit der Verwaltung, bildlich gesprochen den „Gründungsauftrag“ der kommunalen Behörde, in den Blick zu nehmen.

b) Der zweite Schritt wäre dann, sich die Wirklichkeit nicht länger von einer kommunalen „Handeln-wie-gewohnt-Sichtweise“ diktieren zu lassen, sondern sich an den betreffenden Menschen, den Einwanderern und ihren Wünschen und Hoffnungen orientierend zu überlegen, wie kommunales Handeln ausgerichtet werden müsste. Nützlich wäre auch, sich einschlägiger Positionen aus der Zivilgesellschaft zu vergewissern und auf die Systemumwelt neu abzustimmen.

c) Die soziale Intervention orientiert sich auf diese Weise an einer gewissermaßen fiktiven Verwaltungslogik, d.h. sie muss vielmehr das, woran sie sich zu orientieren hat, im Interesse einer guten Orientierung erst erfinden. Die dabei entstehenden Verfahrensweisen könnten dann auch dazu dienen, der Verwaltung neue Wege aufzuzeigen. Insofern wäre das praktische Handeln immer auch kritisch-politisch orientiert.

Das Beispiel zeigt, dass die Pädagogik, die Sozialpädagogik und vor allem auch die Soziale Arbeit in ,wohlgeordneten“ institutionellen Kontexten nicht nur anders, sondern vor allem auch mehr als in den anderen Kontexten herausgefordert sind. Das gilt sicherlich hinsichtlich der Ausrichtung sozialer Intervention ganz besonders, weil es hier an einem angemessenen Andockpartner fehlt. Es gilt aber natürlich auch für andere Praxisformate einer engagierten Sozialen Arbeit. Sie dürfte es in jedem Fall einfacher haben, wenn sie eine ausreichende institutionelle Distanz gegenüber den kommunalen Instanzen einzuhalten vermag.

Die Soziale Arbeit ist anders gefordert. Für sie ist fundamental, sich in der Stadtgesellschaft und der sie bestimmenden Logik wirklich gut auszukennen. Und sie ist ganz besonders gefordert, weil es letztlich darauf ankommt, die „wohlgeordneten“ urbanen Systeme zu mobilisieren und hier die Positionen zu stärken, die die zunehmende Mobilität und Diversität als Teil von urbaner Realität betrachten und bereit sind, sich darauf konstruktiv einzulassen. Erst wenn die „wohlgeordneten“ kommunalen Systeme ausreichend sensibel für den globalgesellschaftlichen Wandel geworden sind, erst dann kann sich die Soziale Arbeit ganz auf ihr Alltagsgeschäft konzentrieren. 


\section{Literaturverzeichnis}

Anhorn, Roland/Stehr, Johannes (2011): Grundmodelle von Gesellschaft und soziale Ausschließung. Zum Gegenstand einer kritischen Forschungsperspektive in der Sozialen Arbeit, in: Elke Schimpf/Johannes Stehr (Hg.): Kritisches Forschen in der Sozialen Arbeit, Wiesbaden, S. 57-76

Beck, Ulrich/Willms, Johannes (2001): Freiheit oder Kapitalismus. Gesellschaft neu denken, Frankfurt am Main

Bommes, Michael/Scherr, Albert (2012): Soziologie der Sozialen Arbeit. Eine Einführung in Formen und Funktionen organisierter Hilfe, Weinheim-Basel

Bukow, Wolf-D./Lösch, Bettina/Ottersbach, Markus/Preißing, Sonja (2013): Partizipation in der Einwanderungsgesellschaft, Wiesbaden

Bukow, Wolf-D./Nikodem, Claudia/Schulze, Erika/Yildiz, Erol (2001): Die multikulturelle Stadt. Von der Selbstverständlichkeit im städtischen Alltag, Opladen

Bukow, Wolf-D./Preißing, Sonja (2010): ,Wir sind kölsche Jungs“. Die ,Kalker Revolte'. Der Kampf um Partizipation in der urbanen Gesellschaft, in: Angela Pilch Ortega/Andrea Felbinger/Regina Mikula/Rudolf Egger (Hg.): Macht-EigensinnEngagement. Lernprozesse gesellschaftlicher Teilhabe, Wiesbaden, S. 145-172

Bukow, Wolf-D./Spindler, Susanne (2011): Fallstricke einer biographischen Hinwendung zum Subjekt in Forschungsprozessen, in: Elke Schimpf/Johannes Stehr (Hg.): Kritisches Forschen in der Sozialen Arbeit, Wiesbaden, S. 275-289

Bukow, Wolf-D. (2010a): Urbanes Zusammenleben. Zum Umgang mit Migration und Mobilität in europäischen Stadtgesellschaften, Wiesbaden

Bukow, Wolf-D. (2010b): Vielfalt in der Postmodernen Stadtgesellschaft. Eine Ortsbestimmung, in: Wolf-D. Bukow (Hg.): Neue Vielfalt in der urbanen Stadtgesellschaft, Wiesbaden, S. 207-232

Clarke, Adele E./Keller, Reiner/Sarnes, Juliane (2010): Situationsanalyse, Wiesbaden

Goffman, Erving (1973): Interaktion. Spaß am Spiel, Rollendistanz, München

Heide, Angela (Hg.) (2010): Aufbruch in die Nähe, Wien, Lerchenfelder Straße. Mikrogeschichten zwischen Lokalidentitäten und Globalisierung, Wien

Hess, Sabine (2009): No integration?! Kulturwissenschaftliche Beiträge zur Integrationsdebatte in Europa, Bielefeld

Müller, Michael (2010): Straßen und Integration, in: Angela Heide (Hg.) (2010): Aufbruch in die Nähe, Wien, Lerchenfelder Straße. Mikrogeschichten zwischen Lokalidentitäten und Globalisierung, Wien, S. 29-54

Pilch Ortega, Angela/Felbinger, Andrea/Mikula, Regina/Egger, Rudolf (Hg.) (2010): Macht-Eigensinn-Engagement. Lernprozesse gesellschaftlicher Teilhabe, Wiesbaden

Pries, Ludger (2010): Transnationalisierung. Theorie und Empirie grenzüberschreitender Vergesellschaftung, Wiesbaden

Pries, Ludger/Sezgin, Zeynep (2010): Jenseits von ,Identität' oder ,Integration“. Grenzen überspannende Migrantenorganisationen, Wiesbaden

Saunders, Doug (2011): Arrival city, London 
Schimpf, Elke/Stehr, Johannes (Hg.) (2011): Kritisches Forschen in der Sozialen Arbeit. Gegenstandsbereiche-Kontextbedingungen-Positionierungen-Perspektiven, Wiesbaden

Senol, Ekrem (2011): Selbstbewusstsein. Eine Gebrauchsanweisung, in: Hilal Sezgin (Hg.): Manifest der Vielen. Deutschland erfindet sich neu, Berlin, S. 53-62

Sezgin, Hilal (Hg.) (2011): Manifest der Vielen. Deutschland erfindet sich neu, Berlin 